\title{
MOLECULAR LINE OBSERVATIONS OF INFRARED DARK CLOUDS: SEEKING THE PRECURSORS TO INTERMEDIATE AND MASSIVE STAR FORMATION
}

\author{
S. E. Ragan, ${ }^{1}$ E. A. Bergin, ${ }^{1}$ R. Plume, ${ }^{2}$ D. L. Gibson, ${ }^{2}$ D. J. Wilner, ${ }^{3}$ S. O’Brien, ${ }^{4}$ and E. Hails ${ }^{5}$ \\ Received 2006 March 25; accepted 2006 June 6
}

\begin{abstract}
We have identified 41 infrared dark clouds from the $8 \mu \mathrm{m}$ maps of the Midcourse Space Experiment (MSX), selected to be found within $1 \mathrm{deg}^{2}$ areas centered on known ultracompact $\mathrm{H}$ II regions. We have mapped these infrared dark clouds in $\mathrm{N}_{2} \mathrm{H}^{+} 1 \rightarrow 0, \mathrm{CS} 2 \rightarrow 1$, and $\mathrm{C}^{18} \mathrm{O} 1 \rightarrow 0$ emission using the Five College Radio Astronomy Observatory. The maps of the different species often show striking differences in morphologies, indicating differences in evolutionary state and/or the presence of undetected, deeply embedded protostars. We derive an average mass for these clouds using $\mathrm{N}_{2} \mathrm{H}^{+}$column densities of $\approx 2500 M_{\odot}$, a value comparable to that found in previous studies of high-mass star-forming cores using other mass tracers. The line widths of these clouds are typically $\sim 2.0-2.9 \mathrm{~km} \mathrm{~s}^{-1}$. Based on the fact that they are dark at $8 \mu \mathrm{m}$, compact, and massive, and have large velocity dispersions, we suggest that these clouds may be the precursor sites of intermediate- and high-mass star formation.
\end{abstract}

Subject headings: ISM: clouds - ISM: molecules — stars: formation

Online material: machine-readable tables

\section{INTRODUCTION}

The study of star formation has made tremendous strides over the past two decades. Advances in observational capabilities have allowed a number of phases of the star formation process to be identified and characterized, starting with the centrally concentrated core of molecular gas that collapses to form a star surrounded by a protoplanetary disk. It has been the isolation of such objects that have not yet formed stars - prestellar cores - that has allowed us to probe the earliest initial stages of star formation (see André et al. 2000; Alves et al. 2001). Most of the progress has focused on the formation of low-mass stars, predominantly because these objects can form isolated from other nearby stars that reduces confusion, and, conveniently, there is a large sample of such objects located in nearby clouds. However, it has now been recognized that most stars that are more massive than the Sun do not form in isolated fashion, but rather in clusters of $>100$ stars (Zinnecker et al. 1993).

Progress in our understanding of high-mass star formation has been hampered by a number of factors: (1) timescales for massive star formation are short and examples in a given state are intrinsically rare, (2) the large distances to the giant molecular clouds (GMCs) that are the birth sites of massive stars make studies of individual objects difficult, and (3) the very nature of clustered star formation increases confusion (cf. Garay \& Lizano 1999). As a result, the objects in the earliest phases - the "prestellar massive cores"- have been difficult to identify.

The traditional method of locating low-mass cores has been to examine optical plates for regions of obscured starlight and then to pursue follow-up molecular line observations (Myers \& Benson 1983; Lee \& Myers 1999). A comparison with the IRAS point source catalog then denotes whether these cores are associated with newly formed stars (Beichman et al. 1986). This

\footnotetext{
1 Department of Astronomy, 500 Church Street, University of Michigan, Ann Arbor, MI 48109.

2 Department of Physics and Astronomy, University of Calgary, Calgary, AB, Canada.

${ }^{3}$ Harvard-Smithsonian Center for Astrophysics, Cambridge, MA.

4 Department of Physics, University of Notre Dame, Notre Dame, IN.

5 Arizona Radio Observatory, Tucson, AZ.
}

method cannot be applied to massive star-forming regions since the greater distances makes isolating individual objects difficult. Moreover, the size and high column densities of GMCs makes it impossible to use optical plates to find individual objects. An analogous method of searching for molecular cores is to search for obscured regions in Galactic mid-infrared background. However, due to atmospheric constraints, ground-based observations in the mid-IR are difficult to obtain. The ISOCAM instrument on the Infrared Space Observatory was used in this fashion, but only with pointed observations toward previously identified cores (e.g., Bacmann et al. 2000).

The Midcourse Space Experiment (MSX) surveyed the Galactic plane in mid-infrared bands spanning from 7 to $25 \mu \mathrm{m}$. This survey revealed a large population of dark clouds, predominantly located toward the inner Galaxy (Egan et al. 1998). Follow-up molecular studies of a few objects confirmed that the obscured regions represent a new population of dense, $n\left(\mathrm{H}_{2}\right)>$ $10^{5} \mathrm{~cm}^{-3}$, and cold, $T<20 \mathrm{~K}$, molecular clouds (Carey et al. 1998). Further comparison to IRAS images demonstrated that most of these clouds are dark from 7 to $100 \mu \mathrm{m}$, presumably because these objects either do not contain newly formed stars, or any newly formed stars are very deeply embedded. As such, a subsample of these objects may trace massive prestellar cores. There has been substantial activity in this field recently, with numerous groups analyzing various samples of infrared dark clouds (IRDCs). These studies have shown that it is likely that IRDCs are the birth sites of high-mass stars and stellar clusters (Sridharan et al. 2005; Menten et al. 2005; Rathborne et al. 2006; Pillai et al. 2006).

We have identified a sample of infrared dark clouds and searched them for emission from the $\mathrm{N}_{2} \mathrm{H}^{+} 1 \rightarrow 0$, CS $2 \rightarrow 1$, and $\mathrm{C}^{18} \mathrm{O} 1 \rightarrow 0$ transitions ( $(2)$. In most cases, we find that the emission closely corresponds to the $M S X$ dark regions. Using a gas temperature of $T=15 \mathrm{~K}$ based on $\mathrm{CO} 1 \rightarrow 0$ data, we deduce several properties of the dark clouds including column density and mass (§3). We then summarize our findings and the implications $(\S 4)$.

\section{SOURCE SELECTION AND OBSERVATIONS}

To search for prestellar massive cores we have compiled a catalog of $M S X$ dark clouds. This catalog is biased, as we have 
TABLE 1

Basic Properties of the Target $M S X$ Pre-Stellar Cores

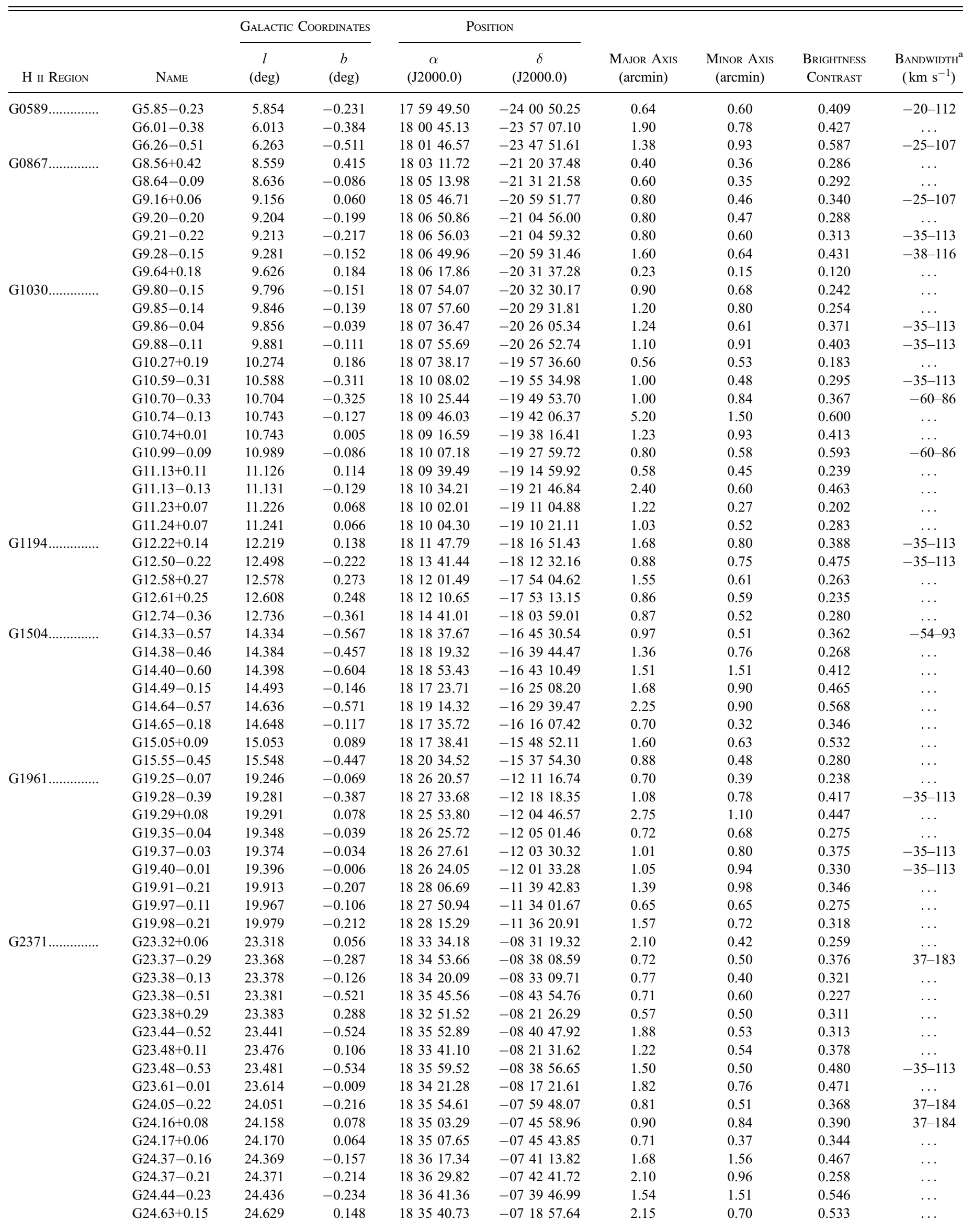


TABLE 1-Continued

\begin{tabular}{|c|c|c|c|c|c|c|c|c|c|}
\hline \multirow[b]{2}{*}{ H II REGION } & \multirow[b]{2}{*}{ NAME } & \multicolumn{2}{|c|}{ Galactic Coordinates } & \multicolumn{2}{|c|}{ Position } & \multirow[b]{2}{*}{$\begin{array}{c}\text { MAJOR AxIS } \\
\text { (arcmin) }\end{array}$} & \multirow[b]{2}{*}{$\begin{array}{l}\text { MINOR AxIS } \\
\text { (arcmin) }\end{array}$} & \multirow[b]{2}{*}{$\begin{array}{c}\text { BRIGHTNESS } \\
\text { CONTRAST }\end{array}$} & \multirow[b]{2}{*}{$\begin{array}{c}\text { BANDWIDTH }^{\mathrm{a}} \\
\left(\mathrm{km} \mathrm{s}^{-1}\right)\end{array}$} \\
\hline & & $\begin{array}{c}l \\
(\operatorname{deg})\end{array}$ & $\begin{array}{c}b \\
(\mathrm{deg})\end{array}$ & $\begin{array}{c}\alpha \\
(\mathrm{J} 2000.0)\end{array}$ & $\begin{array}{c}\delta \\
(\mathrm{J} 2000.0)\end{array}$ & & & & \\
\hline \multirow[t]{5}{*}{ G2572............... } & $\mathrm{G} 25.08+0.20$ & 25.076 & 0.199 & 183619.50 & -065344.33 & 0.80 & 0.63 & 0.363 & $\ldots$ \\
\hline & G25.24-0.22 & 25.243 & -0.216 & 183807.16 & -065616.48 & 0.72 & 0.56 & 0.298 & $\ldots$ \\
\hline & $\mathrm{G} 25.25-0.24$ & 25.248 & -0.236 & 183812.01 & -065633.53 & 1.00 & 0.50 & 0.283 & $\ldots$ \\
\hline & $\mathrm{G} 25.61+0.24$ & 25.613 & 0.239 & 183710.53 & -062401.14 & 1.70 & 1.70 & 0.374 & $\ldots$ \\
\hline & G25.99-0.06 & 25.985 & -0.057 & 183855.26 & -061220.39 & 1.03 & 0.80 & 0.337 & $-35-113$ \\
\hline \multirow[t]{7}{*}{ G2996.............. } & G30.14-0.07 & 30.135 & -0.069 & 184634.71 & -023113.90 & 1.40 & 0.60 & 0.287 & $-35-113$ \\
\hline & G30.31-0.28 & 30.309 & -0.282 & 184739.31 & -022746.38 & 0.82 & 0.63 & 0.226 & $\ldots$ \\
\hline & G30.49-0.39 & 30.494 & -0.392 & 184823.10 & -022054.35 & 0.84 & 0.81 & 0.414 & $22-168$ \\
\hline & G30.53-0.27 & 30.353 & -0.272 & 184742.00 & -022508.99 & 1.25 & 0.91 & 0.310 & $-35-113$ \\
\hline & G30.58-0.25 & 30.579 & -0.252 & $1848 \quad 02.48$ & -021232.12 & 1.53 & 0.58 & 0.305 & $\ldots$ \\
\hline & G30.66+0.05 & 30.663 & 0.046 & 184708.00 & -015953.60 & 1.82 & 0.80 & 0.358 & $\ldots$ \\
\hline & G30.69+0.06 & 30.689 & 0.056 & 184708.72 & -015813.85 & 0.75 & 0.63 & 0.217 & $\ldots$ \\
\hline \multirow[t]{8}{*}{ G3141............... } & G30.89+0.14 & 30.894 & 0.136 & 184714.08 & -014505.75 & 1.60 & 0.60 & 0.321 & $-35-113$ \\
\hline & G30.98-0.15 & 30.978 & -0.148 & 184823.97 & -014823.07 & 1.33 & 0.80 & 0.411 & $-35-113$ \\
\hline & G31.02-0.12 & 31.024 & -0.116 & 184822.17 & -014503.19 & 1.62 & 0.70 & 0.450 & $-35-113$ \\
\hline & G31.23+0.02 & 31.226 & 0.024 & 185003.85 & -003702.40 & 1.20 & 0.80 & 0.361 & $\ldots$ \\
\hline & G31.39+0.30 & 31.391 & 0.296 & 184734.34 & -011410.89 & 2.10 & 1.70 & 0.349 & $\ldots$ \\
\hline & G31.70-0.50 & 31.699 & -0.496 & 185057.27 & -011924.42 & 0.68 & 0.50 & 0.348 & $\ldots$ \\
\hline & G31.71-0.49 & 31.723 & -0.486 & 185057.76 & -011751.09 & 0.63 & 0.60 & 0.336 & $\ldots$ \\
\hline & G32.01+0.05 & 32.013 & 0.056 & 184933.70 & -004732.25 & 1.17 & 1.10 & 0.526 & $-35-113$ \\
\hline \multirow[t]{4}{*}{ G3350............... } & G32.84-0.03 & 32.843 & -0.032 & 185123.35 & -000537.42 & 1.30 & 0.97 & 0.357 & $\ldots$ \\
\hline & G33.36-0.01 & 33.363 & -0.007 & 185214.91 & 002249.67 & 1.58 & 0.84 & 0.288 & $\ldots$ \\
\hline & G33.42+0.13 & 33.418 & 0.126 & 185152.52 & 002924.18 & 0.44 & 0.25 & 0.217 & $\ldots$ \\
\hline & G33.70-0.02 & 33.699 & -0.016 & 185253.60 & 004031.43 & 1.80 & 0.50 & 0.250 & $\ldots$ \\
\hline \multirow[t]{6}{*}{ G3426............. } & G33.82-0.22 & 33.819 & -0.219 & 185350.10 & 004122.54 & 0.90 & 0.53 & 0.432 & $2-149$ \\
\hline & G34.13+0.08 & 34.134 & 0.076 & 185321.57 & 010616.14 & 1.68 & 0.50 & 0.254 & $\ldots$ \\
\hline & G34.26+0.19 & 34.263 & 0.189 & 185311.55 & 011614.93 & 1.75 & 0.61 & 0.271 & $\ldots$ \\
\hline & G34.74-0.12 & 34.739 & -0.122 & 185510.11 & 013309.25 & 0.90 & 0.80 & 0.393 & $-15-131$ \\
\hline & G34.74+0.01 & 34.744 & 0.006 & 185443.32 & 013655.46 & 0.75 & 0.57 & 0.212 & $\ldots$ \\
\hline & G35.04-0.47 & 35.043 & -0.474 & 185658.62 & 013944.74 & 0.65 & 0.41 & 0.312 & $\ldots$ \\
\hline \multirow[t]{4}{*}{ G3520.............. } & G34.63-1.03 & 34.627 & -1.026 & 185810.95 & 010225.33 & 0.26 & 0.18 & 0.447 & $-29-117$ \\
\hline & G34.78-0.80 & 34.778 & -0.804 & 185740.08 & 011633.76 & 0.56 & 0.45 & 0.341 & $-35-114$ \\
\hline & G35.02-1.50 & 35.018 & -1.497 & 190034.38 & 011023.16 & 0.97 & 0.35 & 0.375 & $\ldots$ \\
\hline & G35.20-0.72 & 35.203 & -0.721 & 185808.92 & 014131.31 & 0.78 & 0.51 & 0.436 & $-29-117$ \\
\hline \multirow[t]{5}{*}{ G3755............. } & G37.08-0.15 & 37.081 & -0.149 & 185932.80 & 033725.91 & 0.42 & 0.40 & 0.321 & $\ldots$ \\
\hline & G37.25+0.01 & 37.253 & 0.011 & 185917.48 & 035059.94 & 0.98 & 0.52 & 0.296 & $\ldots$ \\
\hline & G37.42+0.17 & 37.418 & 0.173 & 185900.95 & 040414.78 & 0.70 & 0.64 & 0.342 & $\ldots$ \\
\hline & G37.44+0.14 & 37.439 & 0.138 & 185910.75 & 040424.37 & 0.79 & 0.40 & 0.322 & $-35-113$ \\
\hline & G37.89-0.15 & 37.886 & -0.152 & 190102.00 & 042018.23 & 1.03 & 0.58 & 0.325 & $-26-107$ \\
\hline \multirow[t]{3}{*}{ G4318................ } & G43.19-0.16 & 43.187 & -0.162 & 191053.01 & 090230.58 & 0.42 & 0.20 & 0.304 & $\ldots$ \\
\hline & G43.32-0.20 & 43.318 & -0.204 & 191116.78 & 090818.83 & 0.50 & 0.42 & 0.165 & $\ldots$ \\
\hline & G43.78+0.05 & 43.776 & 0.046 & 191114.38 & 093936.89 & 0.92 & 0.91 & 0.377 & $-23-124$ \\
\hline G4389............... & G43.64-0.82 & 43.644 & -0.824 & 191407.05 & 090824.64 & 0.60 & 0.21 & 0.577 & $-19-127$ \\
\hline G4426................. & G44.29-0.09 & 44.291 & -0.092 & 191242.25 & 100310.40 & 0.71 & 0.46 & 0.219 & $\ldots$ \\
\hline \multirow[t]{3}{*}{ G5023 „............... } & G48.84+0.15 & 48.836 & 0.151 & 192029.90 & 141112.12 & 1.22 & 0.58 & 0.317 & $\ldots$ \\
\hline & $\mathrm{G} 48.84+0.14$ & 48.848 & 0.136 & 192034.57 & 141124.84 & 0.70 & 0.43 & 0.242 & $\ldots$ \\
\hline & G50.07+0.06 & 50.071 & 0.059 & 192314.36 & 151358.11 & 0.86 & 0.33 & 0.378 & $-13-133$ \\
\hline G5031................ & G51.00-0.18 & 51.001 & -0.177 & 192255.92 & 155624.24 & 0.60 & 0.35 & 0.290 & $\ldots$ \\
\hline G5410................ & G53.88-0.18 & 53.879 & -0.181 & 193142.73 & 182755.78 & 1.12 & 0.42 & 0.417 & $-30-116$ \\
\hline G6148................ & G61.52+0.02 & 61.519 & 0.024 & 194709.72 & 251300.46 & 0.60 & 0.45 & 0.334 & $\ldots$ \\
\hline G7578............. & G75.75+0.75 & 75.753 & 0.749 & 201957.75 & 373901.90 & 1.10 & 0.90 & 0.474 & $-33-113$ \\
\hline & G76.38+0.63 & 76.381 & 0.626 & 202217.04 & 380550.23 & 0.40 & 0.30 & 0.318 & $-33-113$ \\
\hline
\end{tabular}

Noте.-Units of right ascension are hours, minutes, and seconds, and units of declination are degrees, arcminutes, and arcseconds. Table 1 is also available in machine-readable form in the electronic edition of the Astrophysical Journal Supplement.

${ }^{\text {a }}$ For observed regions, we give the total velocity range to which the observations were sensitive. A given object was probed in the same velocity range for each observed transition.

only searched the released $M S X$ band A (centered at $8.8 \mu \mathrm{m}$ ) images for infrared dark clouds in the vicinity of known ultracompact (UC) H II regions from the Wood \& Churchwell (1989) catalog. More specifically, we searched for absorbing clouds within a square degree centered on a given UC H II region. This strategy takes advantage of the fact that young stars generally form in clusters, and, therefore, a good place to search for the precursors to massive stars is in the vicinity of regions with current massive star formation evidenced by the UC H II regions. In this fashion we have isolated 114 infrared dark clouds, of which only a small fraction (15\%) have known associations with radio sources or masers. From this catalog of 114 infrared dark 
clouds we selected a subsample of the 41 most compact and most opaque $\left(\tau_{8.8 \mu \mathrm{m}} \gtrsim 0.4\right)$ cores and targeted these for molecular line observations.

We mapped 41 of the sample of $M S X$ dark clouds in emission from $\mathrm{C}^{18} \mathrm{O} J=1 \rightarrow 0(\nu=109.782 \mathrm{GHz}), \mathrm{CS} J=2 \rightarrow 1$ $(\nu=97.981 \mathrm{GHz})$, and $\mathrm{N}_{2} \mathrm{H}^{+} J=1 \rightarrow 0(\nu=93.173 \mathrm{GHz})$ using the $14 \mathrm{~m}$ Five College Radio Astronomy Observatory (FCRAO). The observations were made in 2002 February, May, and December using the 16 element focal plane array receiver SEQUOIA. Each 2. $5 \times 2$. 5 region was mapped with the $50^{\prime \prime}$ beam, with typical rms noise levels of $\sim 0.05-0.1 \mathrm{~K}$. We used the Narrow Band Correlator configured to a velocity resolution of $\sim 0.13 \mathrm{~km} \mathrm{~s}^{-1}$. Typical system temperatures $\left(T_{\text {sys }}\right)$ were $200-$ $300 \mathrm{~K}$. Main beam efficiencies $\left(\eta_{\mathrm{mb}}\right)$ were approximated at $50 \%$ from the standard FCRAO values. This is accurate within a few percent for each transition. For each spectrum, a first-order, linear baseline was fitted to remove instrumental and continuum offsets and drift.

This selection of species is motivated by studies of low-mass prestellar clouds. $\mathrm{N}_{2} \mathrm{H}^{+}$is unlikely to significantly suffer from the effects of depletion as the core condenses, and this species is a good tracer of the dense centers of starless cores (Bergin \& Langer 1997; Tafalla et al. 2002). Conversely, $\mathrm{C}^{18} \mathrm{O}$ and $\mathrm{CS}$ emission can be used to trace the outer layers. Table 1 lists some basic dark cloud properties such as the $\mathrm{H}$ II region name, the coordinates, the size, and the center-to-edge brightness contrast. The brightness contrast was obtained by comparing the brightness in the band centered at $8.8 \mu \mathrm{m}$ at the center of the core with the average background brightness, estimated from an average of the intensities in a vertical and horizontal slit across the dark region. If the dark cloud was searched for molecular emission, we provide the LSR velocity range to which the observations were sensitive.

In an Appendix, we provide a source by source description of any associations with known star formation indicators (e.g., $I R A S$ sources, masers, radio continuum sources). The large majority of our sources have no association with any known strong infrared source.

\section{RESULTS}

\subsection{Molecular Line Fits}

Each line is fitted with a Gaussian profile to determine the integrated intensity, line width, and the LSR velocity of the emitting material. Table 2 presents the results of the spectral line fitting. All parameters were extracted by standard Gaussian fitting methods in the CLASS package (Buisson et al. 2002); for $\mathrm{N}_{2} \mathrm{H}^{+}$, the seven main hyperfine components were fitted together using the HFS routine. The reported line-center velocity corresponds to that of the strongest hyperfine component $\left(J=1 \rightarrow 0, F_{1}, F=\right.$ $2,3 \rightarrow 1,2)$ at $93.1738 \mathrm{GHz}$.

Our observations were obtained with velocity resolution of $0.13 \mathrm{~km} \mathrm{~s}^{-1}$, and to increase the signal to noise, we on occasion smoothed the line profiles by a factor of 2 . As such, we had little sensitivity to structure within the line. Within our sensitivity limits (see $\S 2$ ), we see no evidence for extended line wings, and all lines were well fitted by single Gaussians. We report here only basic line properties.

\subsection{Molecular Emission Morphologies}

Figure 1 displays the $M S X$ images of each dark cloud overlaid with the integrated intensity maps of molecular emission for all observed molecular transitions. The contour levels for each dark cloud are specified in the captions. In some cases, multiple velocity components are detected, and the different velocity components are given different colors. The blue contours always correspond to the molecular emission morphology that most closely corresponds to the distribution of $8 \mu \mathrm{m}$ absorption seen in the MSX image and, therefore, is most likely to be associated with the dark cloud. The line properties of the other velocity components (i.e., emission that is unassociated with the absorbing cloud) are not reported. There are three cases in which an $\mathrm{a} / \mathrm{b}$ designation was assigned to distinguish two emission peaks. For G14.33-0.57 and G23.48-0.53, there are two spatially distinct and separate emission peaks at approximately the same characteristic velocity. This may suggest that in these cases, we were able to resolve spatially separated fragments of a cloud. In the case of G37.44+ 0.14 , there are two velocity components that appear to correspond to absorbing regions (the $40 \mathrm{~km} \mathrm{~s}^{-1}$ feature corresponding to the central absorbing cloud; the $18 \mathrm{~km} \mathrm{~s}^{-1}$ feature corresponding to absorption to the north and west). Based on our assumptions, this would indicate that there are two unassociated dark clouds apparent in the same region of the sky by chance.

The morphological differences seen in Figure 1 are striking. Some maps show well defined cores in all three molecular tracers (e.g., G32.01+0.05). Other maps, like G06.26-0.51, show fairly well defined cores in $\mathrm{C}^{18} \mathrm{O}$ and $\mathrm{CS}$, but nothing obvious is seen in the $\mathrm{N}_{2} \mathrm{H}^{+}$. And others, like G09.21-0.22, show a strong centrally concentrated $\mathrm{N}_{2} \mathrm{H}^{+}$core but $\mathrm{CS}$ that is much more diffuse. Finally, there are cores like G10.59-0.31 that show no real evidence for molecular emission centered on the dark cloud at all. In this case, it is possible that any molecular emission directly associated with the dark cloud lies outside of the observed velocity band (see Table 1).

These differences in the molecular emission maps may be the result of differences in the evolutionary states. It is well known that $\mathrm{CS}$ and $\mathrm{C}^{18} \mathrm{O}$ can form relatively quickly in the gas phase, whereas $\mathrm{N}_{2} \mathrm{H}^{+}$takes significantly longer. However, as density enhances, the $\mathrm{CS}$ and $\mathrm{C}^{18} \mathrm{O}$ tend to deplete onto the surfaces of dust grains, whereas $\mathrm{N}_{2} \mathrm{H}^{+}$will remain in the gas phase. On protostar formation, $\mathrm{CS}$ and $\mathrm{C}^{18} \mathrm{O}$ can be released from dust grain surfaces (e.g., see models of Bergin \& Langer 1997; Lee et al. 2004). Therefore, two scenarios can lead to the low abundance of $\mathrm{N}_{2} \mathrm{H}^{+}$relative to $\mathrm{CS}$ or $\mathrm{C}^{18} \mathrm{O}$ : the star-forming core is at an early stage of condensation and the densities are low such that $\mathrm{CS}$ and $\mathrm{C}^{18} \mathrm{O}$ would be not affected by dust depletion, or the presence of a protostar has released the $\mathrm{CS}$ and $\mathrm{C}^{18} \mathrm{O}$ from dust grains, all the while the $\mathrm{N}_{2} \mathrm{H}^{+}$abundance is essentially unchanged throughout the process. Another possible explanation of the differences seen in the maps is that some of the dark clouds may contain as yet undetected protostars, obscured by the high opacity apparent in the $8.8 \mu \mathrm{m} \mathrm{MSX}$ images. If a protostar is present, it can alter the local gas chemistry via grain mantle evaporation, which can change the emission morphologies (Jørgensen 2004). We are using data from the Spitzer Space Telescope to determine which dark clouds are truly starless and which contain embedded protostars in a subsample of these objects (S. E. Ragan et al. 2006 , in preparation).

\subsection{Distance Estimates}

The kinematic distance to each dark cloud is calculated using the line center velocity and the Milky Way rotation curve model of Fich et al. (1989). The distance assignments are presented in Table 3 for dark clouds for which we estimated masses. (Sources to which we are unable to assign a distance or those that show no significant emission are not subject to further calculations.) For every position, there is both a "near" and "far" distance solution that corresponds to the characteristic velocity of the emission. In 
TABLE 2

Molecular Line Observations

\begin{tabular}{|c|c|c|c|c|c|c|c|c|c|c|c|}
\hline \multirow[b]{2}{*}{ Source } & \multirow[b]{2}{*}{$\begin{array}{c}\Delta \alpha \\
(\operatorname{arcmin})\end{array}$} & \multirow[b]{2}{*}{$\begin{array}{c}\Delta \delta \\
(\operatorname{arcmin})\end{array}$} & \multicolumn{3}{|c|}{$\mathrm{N}_{2} \mathrm{H}^{+} J=1-0$} & \multicolumn{3}{|c|}{$\mathrm{C}^{18} \mathrm{O} J=1-0$} & \multicolumn{3}{|c|}{$\operatorname{CS} J=2-1$} \\
\hline & & & $\begin{array}{c}\int T_{A}^{*} d v \\
\left(\mathrm{~K} \mathrm{~km} \mathrm{~s}^{-1}\right)\end{array}$ & $\begin{array}{c}V \\
\left(\mathrm{~km} \mathrm{~s}^{-1}\right)\end{array}$ & $\begin{array}{c}\Delta v \\
\left(\mathrm{~km} \mathrm{~s}^{-1}\right)\end{array}$ & $\begin{array}{c}\int T_{A}^{*} d v \\
\left(\mathrm{~K} \mathrm{~km} \mathrm{~s}^{-1}\right)\end{array}$ & $\begin{array}{c}V \\
\left(\mathrm{~km} \mathrm{~s}^{-1}\right)\end{array}$ & $\begin{array}{c}\Delta v \\
\left(\mathrm{~km} \mathrm{~s}^{-1}\right)\end{array}$ & $\begin{array}{c}\int T_{A}^{*} d v \\
\left(\mathrm{~K} \mathrm{~km} \mathrm{~s}^{-1}\right)\end{array}$ & $\begin{array}{c}V \\
\left(\mathrm{~km} \mathrm{~s}^{-1}\right)\end{array}$ & $\begin{array}{c}\Delta v \\
\left(\mathrm{~km} \mathrm{~s}^{-1}\right)\end{array}$ \\
\hline G05.85-0.23...... & 0.4 & -0.4 & $1.21(0.15)$ & $17.2(0.1)$ & $0.8(0.1)$ & $1.44(0.17)$ & $17.0(0.1)$ & $1.7(0.2)$ & $0.47(0.10)$ & $16.9(0.2)$ & $2.2(0.3)$ \\
\hline G06.26-0.51..... & 0.0 & 0.0 & $(<0.15)$ & $\ldots$ & $\ldots$ & $1.69(0.11)$ & $22.7(0.1)$ & $2.3(0.2)$ & $1.64(0.15)$ & $23.2(0.2)$ & $4.2(0.5)$ \\
\hline G09.16+0.06 ....... & 0.0 & 0.0 & $(<0.12)$ & $\ldots$ & $\ldots$ & $1.39(0.09)$ & $31.3(0.1)$ & $1.7(0.1)$ & $0.40(0.07)$ & $31.3(0.1)$ & $1.2(0.3)$ \\
\hline G09.21-0.22...... & 0.0 & 0.0 & $3.59(0.15)$ & $42.8(0.1)$ & $1.8(0.2)$ & $\ldots$ & $\ldots$ & $\ldots$ & $1.29(0.11)$ & $42.7(0.1)$ & $2.8(0.3)$ \\
\hline 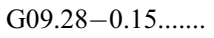 & 0.0 & 0.0 & $3.66(0.10)$ & $41.4(0.1)$ & $1.9(0.4)$ & $\ldots$ & $\ldots$ & $\ldots$ & $1.27(0.12)$ & $41.3(0.1)$ & $2.6(0.3)$ \\
\hline G09.86-0.04...... & 0.0 & 0.0 & $0.87(0.11)$ & $18.1(0.1)$ & $1.1(0.2)$ & $\ldots$ & $\ldots$ & $\ldots$ & $1.52(0.14)$ & $17.8(0.1)$ & $2.3(0.2)$ \\
\hline 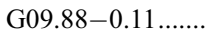 & 0.0 & 0.0 & $(<0.13)$ & $\ldots$ & $\ldots$ & $\ldots$ & $\ldots$ & $\ldots$ & $0.54(0.15)$ & $17.3(0.2)$ & $1.8(0.6)$ \\
\hline 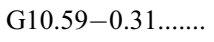 & 0.0 & 0.0 & $(<0.40)$ & $\ldots$ & $\ldots$ & $\ldots$ & $\ldots$ & $\ldots$ & $(<0.24)$ & $\ldots$ & $\ldots$ \\
\hline 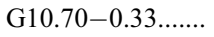 & 0.0 & 0.0 & $(<0.13)$ & $\ldots$ & $\ldots$ & $\ldots$ & $\ldots$ & $\ldots$ & $(<0.13)$ & $\ldots$ & $\ldots$ \\
\hline G10.99-0.09...... & 0.0 & 0.0 & $4.24(0.11)$ & $29.6(0.1)$ & $2.4(0.2)$ & $2.25(0.17)$ & $29.5(0.1)$ & $2.2(0.2)$ & $1.00(0.12)$ & $29.2(0.3)$ & $4.2(0.5)$ \\
\hline G12.22+0.14 ....... & 0.0 & 0.0 & $3.60(0.08)$ & $39.6(0.1)$ & $1.7(0.1)$ & $\ldots$ & $\ldots$ & $\ldots$ & $1.78(0.07)$ & $36.7(0.1)$ & $2.2(0.1)$ \\
\hline 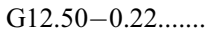 & 0.0 & 0.0 & $2.97(0.10)$ & $35.8(0.1)$ & $1.8(0.1)$ & $1.60(0.13)$ & $35.7(0.1)$ & $1.8(0.2)$ & $1.40(0.10)$ & $35.6(0.1)$ & $2.0(0.2)$ \\
\hline G14.33-0.57a $\ldots .$. & 0.0 & 0.0 & $(<0.14)$ & $\ldots$ & $\ldots$ & $2.19(0.15)$ & $19.3(0.1)$ & $2.1(0.2)$ & $1.25(0.13)$ & $19.6(0.1)$ & $2.4(0.3)$ \\
\hline G14.33-0.57b.... & -1.7 & 0.8 & $1.42(0.14)$ & $20.0(0.1)$ & $1.1(0.2)$ & $1.81(0.12)$ & $19.9(0.1)$ & $1.6(0.2)$ & $0.84(0.10)$ & $20.3(0.1)$ & $1.4(0.2)$ \\
\hline G19.28-0.39 ...... & 0.0 & 0.0 & $(<0.17)$ & $\ldots$ & $\ldots$ & $\ldots$ & $\ldots$ & $\ldots$ & $0.26(0.07)$ & $54.0(0.1)$ & $1.1(0.2)$ \\
\hline G19.37-0.03...... & 0.0 & 0.0 & $3.62(0.11)$ & $27.3(0.1)$ & $2.5(0.1)$ & $\ldots$ & $\ldots$ & $\ldots$ & $3.02(0.07)$ & $27.0(0.1)$ & $3.8(0.1)$ \\
\hline G19.40-0.01...... & 0.0 & 0.0 & $0.95(0.11)$ & $27.0(0.1)$ & $1.2(0.3)$ & $\ldots$ & $\ldots$ & $\ldots$ & $0.81(0.06)$ & $26.5(0.1)$ & $2.9(0.3)$ \\
\hline G23.37-0.29..... & 0.0 & 0.0 & $2.88(0.27)$ & $78.5(0.1)$ & $2.0(0.2)$ & $3.97(0.21)$ & $78.1(0.1)$ & $2.7(0.2)$ & $2.30(0.12)$ & $77.8(0.1)$ & $4.8(0.3)$ \\
\hline G23.48-0.53a.... & 0.0 & 0.0 & $1.45(0.12)$ & $64.8(0.1)$ & $2.5(0.4)$ & $\ldots$ & $\ldots$ & $\ldots$ & $0.90(0.09)$ & $63.9(0.3)$ & $4.8(0.6)$ \\
\hline $\mathrm{G} 23.48-0.53 \mathrm{~b} \ldots .$. & -2.1 & -2.1 & $1.24(0.12)$ & $62.8(0.1)$ & $2.9(0.4)$ & $\ldots$ & $\ldots$ & $\ldots$ & $0.73(0.08)$ & $62.7(0.3)$ & $2.9(0.4)$ \\
\hline $\mathrm{G} 24.05-0.22 \ldots \ldots$. & 0.0 & 0.0 & $2.71(0.21)$ & $81.4(0.1)$ & $1.9(0.3)$ & $2.30(0.10)$ & $81.5(0.1)$ & $2.0(0.1)$ & $1.00(0.13)$ & $82.0(0.2)$ & $2.8(0.5)$ \\
\hline $\mathrm{G} 24.16+0.08 \ldots \ldots$ & 0.0 & 0.0 & $(<0.10)$ & $\ldots$ & $\ldots$ & $1.64(0.16)$ & $51.8(0.1)$ & $1.9(0.2)$ & $(<0.10)$ & $\ldots$ & \\
\hline G25.99-0.06...... & 0.0 & 0.0 & $0.80(0.15)$ & $89.9(0.3)$ & $1.6(0.5)$ & $\ldots$ & $\ldots$ & $\ldots$ & $1.05(0.11)$ & $90.2(0.1)$ & $2.4(0.3)$ \\
\hline 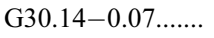 & 0.0 & 0.0 & $(<0.12)$ & $\ldots$ & $\ldots$ & $\ldots$ & $\ldots$ & $\ldots$ & $(<0.15)$ & $86.8(0.2)^{\mathrm{a}}$ & $2.7(0.5)^{\mathrm{a}}$ \\
\hline G30.49-0.39..... & 1.2 & -0.8 & $(<0.14)$ & $\ldots$ & $\ldots$ & $\ldots$ & $\ldots$ & $\ldots$ & $0.62(0.12)$ & $106.4(0.3)$ & $3.0(0.9)$ \\
\hline 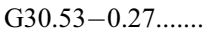 & 0.0 & 0.0 & $(<0.12)$ & $\ldots$ & $\ldots$ & $\ldots$ & $\ldots$ & $\ldots$ & $1.73(0.19)$ & $102.9(0.4)$ & $7.3(0.9)$ \\
\hline G30.89+0.14 ...... & 0.0 & 0.0 & $1.29(0.13)$ & $96.5(0.2)$ & $3.4(0.3)$ & $\ldots$ & $\ldots$ & $\ldots$ & $0.56(0.10)$ & $95.9(0.3)$ & $3.0(0.5)$ \\
\hline 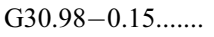 & -0.4 & 0.0 & $4.56(0.12)$ & $77.9(0.1)$ & $2.6(0.1)$ & $\ldots$ & $\ldots$ & $\ldots$ & $2.27(0.11)$ & $77.9(0.1)$ & $4.3(0.2)$ \\
\hline G31.02-0.12 $\ldots \ldots$. & 0.0 & 0.0 & $1.57(0.07)$ & $76.6(0.1)$ & $2.2(0.2)$ & $1.24(0.11)$ & $76.2(0.1)$ & $3.0(0.3)$ & $0.66(0.08)$ & $76.6(0.2)$ & $3.3(0.4)$ \\
\hline G32.01+0.05 ....... & 0.0 & 0.0 & $7.18(0.10)$ & $95.3(0.1)$ & $3.9(0.1)$ & $4.42(0.12)$ & $97.2(0.1)$ & $4.7(0.2)$ & $4.03(0.09)$ & $96.0(0.1)$ & $6.8(0.2)$ \\
\hline G33.82-0.22 ...... & 0.0 & 0.0 & $1.03(0.11)$ & $11.3(0.1)$ & $1.0(0.3)$ & $\ldots$ & $\ldots$ & $\ldots$ & $0.48(0.09)$ & $11.5(0.1)$ & $1.0(0.2)$ \\
\hline 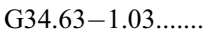 & 0.0 & 0.0 & $1.16(0.15)$ & $13.6(0.2)$ & $2.6(0.6)$ & $0.58(0.08)$ & $12.8(0.1)$ & $1.1(0.2)$ & $(<0.15)$ & $\ldots$ & $\ldots$ \\
\hline 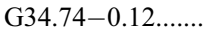 & 0.0 & 0.0 & $1.92(0.17)$ & $79.1(0.1)$ & $2.7(0.3)$ & $3.51(0.14)$ & $78.9(0.2)$ & $2.1(0.1)$ & $1.11(0.10)$ & $78.9(0.2)$ & $3.8(0.4)$ \\
\hline $\mathrm{G} 34.78-0.80 \ldots \ldots$. & 0.0 & 0.0 & $0.33(0.11)$ & $43.2(0.4)$ & $3.0(0.7)$ & $2.25(0.10)$ & $44.1(0.1)$ & $3.3(0.1)$ & $1.83(0.07)$ & $43.5(0.1)$ & $3.0(0.2)$ \\
\hline $\mathrm{G} 35.20-0.72 \ldots \ldots$. & 0.0 & 0.0 & $3.02(0.20)$ & $33.1(0.1)$ & $2.5(0.3)$ & $2.04(0.12)$ & $33.2(0.1)$ & $1.9(0.1)$ & $1.91(0.13)$ & $33.2(0.1)$ & $3.4(0.3)$ \\
\hline G37.44+0.14a $\ldots \ldots$. & 0.0 & 0.0 & $(<0.10)$ & $\ldots$ & $\ldots$ & $1.14(0.06)$ & $40.0(0.1)$ & $1.6(0.1)$ & $0.77(0.04)$ & $40.1(0.1)$ & $1.4(0.1)$ \\
\hline $\mathrm{G} 37.44+0.14 \mathrm{~b} \ldots .$. & -2.5 & -0.4 & $0.65(0.08)$ & $17.8(0.1)$ & $0.5(0.1)$ & $0.52(0.04)$ & $17.8(0.1)$ & $0.7(0.1)$ & $0.25(0.03)$ & $17.7(0.1)$ & $0.9(0.1)$ \\
\hline 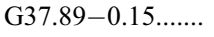 & 0.0 & 0.0 & $0.45(0.08)$ & $12.9(0.1)$ & $0.7(0.1)$ & $0.63(0.04)$ & $12.9(0.1)$ & $0.7(0.1)$ & $0.36(0.04)$ & $13.0(0.1)$ & $0.7(0.1)$ \\
\hline 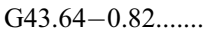 & 0.4 & 2.4 & $(<0.25)$ & $\ldots$ & $\ldots$ & $\ldots$ & $\ldots$ & $\ldots$ & $0.25(0.05)$ & $85.4(0.1)$ & $0.5(0.1)$ \\
\hline G43.78+0.05 $\ldots \ldots .$. & 0.0 & 0.0 & $(<0.17)$ & $\ldots$ & $\ldots$ & $\ldots$ & $\ldots$ & $\ldots$ & $(<0.09)$ & $\ldots$ & $\ldots$ \\
\hline G50.07+0.06 ....... & -0.8 & -0.8 & $(<0.19)$ & $\ldots$ & $\ldots$ & $\ldots$ & $\ldots$ & $\ldots$ & $0.73(0.08)$ & $54.8(0.1)$ & $1.5(0.2)$ \\
\hline G53.88-0.18...... & 0.0 & 0.0 & $(<0.21)$ & $\ldots$ & $\ldots$ & $\ldots$ & $\ldots$ & $\ldots$ & $(<0.12)$ & $\ldots$ & $\ldots$ \\
\hline G75.75+0.75 $\ldots \ldots$. & 0.0 & 0.0 & $(<0.12)$ & $\ldots$ & $\ldots$ & $\ldots$ & $\ldots$ & $\ldots$ & $(<0.07)$ & $\ldots$ & $\ldots$ \\
\hline G76.38+0.63 ...... & 0.0 & 0.0 & $(<0.15)$ & $\ldots$ & $\ldots$ & $\ldots$ & $\ldots$ & $\ldots$ & $(<0.07)$ & $\ldots$ & $\ldots$ \\
\hline
\end{tabular}

Noте.-Table 2 is also available in machine-readable form in the electronic edition of the Astrophysical Journal Supplement.

${ }^{a}$ Nondetection of CS in single scan. Average of 25 scans detects a weak line at the $5 \sigma$ level. The velocity and line width from this average are provided in the table.

addition to this ambiguity, Fich et al. (1989) cite a $\pm 14 \%$ maximal deviation of the data from their rotation curve model; based on this consideration, we calculate errors in the distances and provide them in Table 3. In the cases where no error range (or an incomplete one) is given, no physical solution exists when calculating the distance with that error offset. For all subsequent calculations, we assume that the dark cloud is located at the "near" distance. We believe that this is a reasonable assumption since the clouds are seen in absorption against the Galactic mid-infrared background and, therefore, are unlikely to reside at the "far" distance. Assuming the "near" kinematical distance, which is also listed in Table 4 , a typical core has a diameter of $\sim 0.9 \mathrm{pc}$.
Interestingly, the kinematical distances for the velocity component associated with the absorption are seldom coincident with the distances estimated for the $\mathrm{UC} \mathrm{H}$ II region that was the original search target. In most cases we detect molecular emission at a single velocity, and no possible distance solution from the Galactic rotation curve is consistent with the distance to the $\mathrm{UC} \mathrm{H}$ II region. However, in the cases where there are multiple velocity components, the distance to the $\mathrm{UC} \mathrm{H}$ II region is often consistent with one of the kinematic distance solutions for a secondary velocity component. For example, G37.44+0.14 has a secondary detection of a component at $18 \mathrm{~km} \mathrm{~s}^{-1}$, and the "far" distance associated with it $(12.34 \mathrm{kpc})$ is very close to the distance 

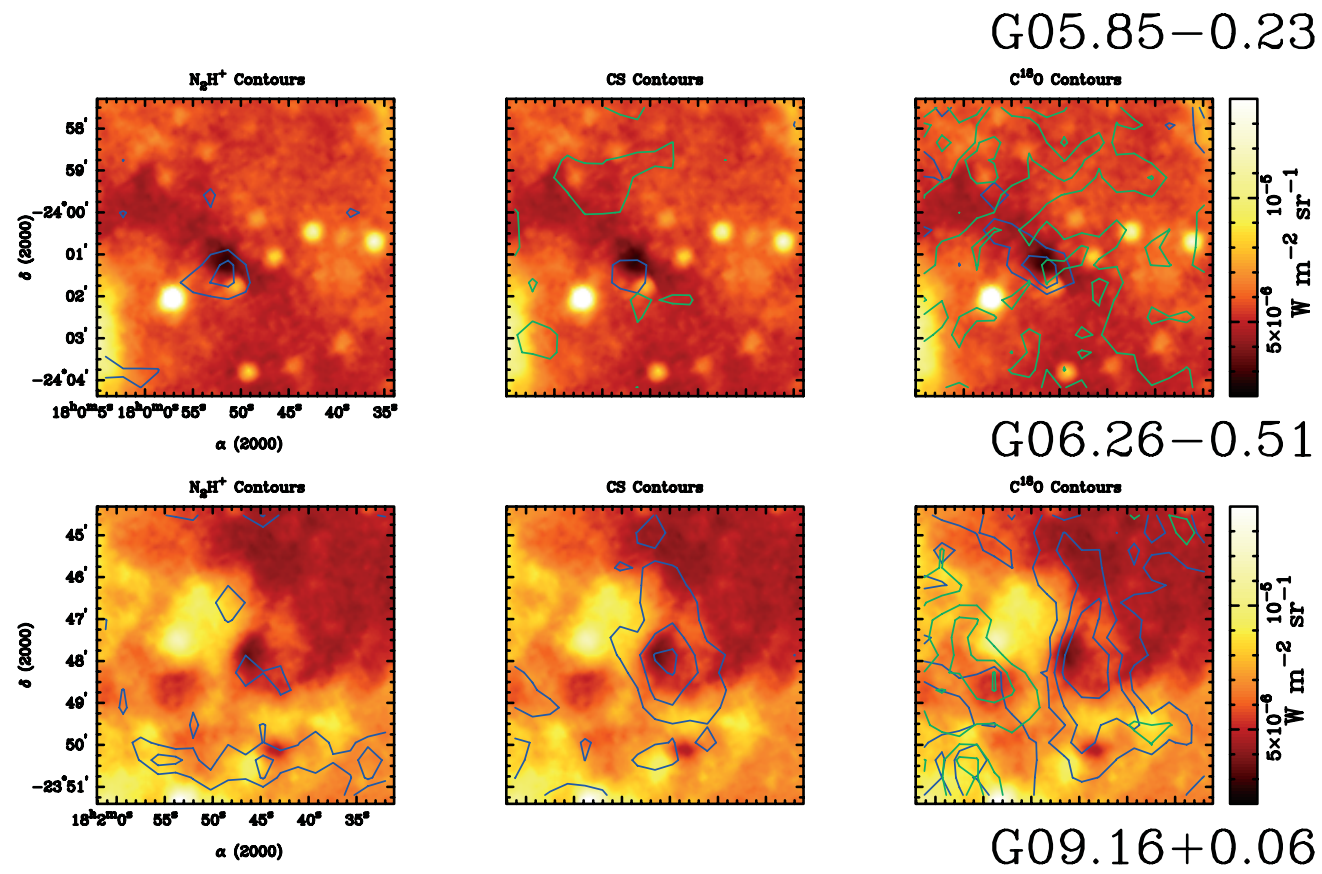

G06.26-0.51

$\mathrm{c}^{180} \mathrm{O}$ Contours
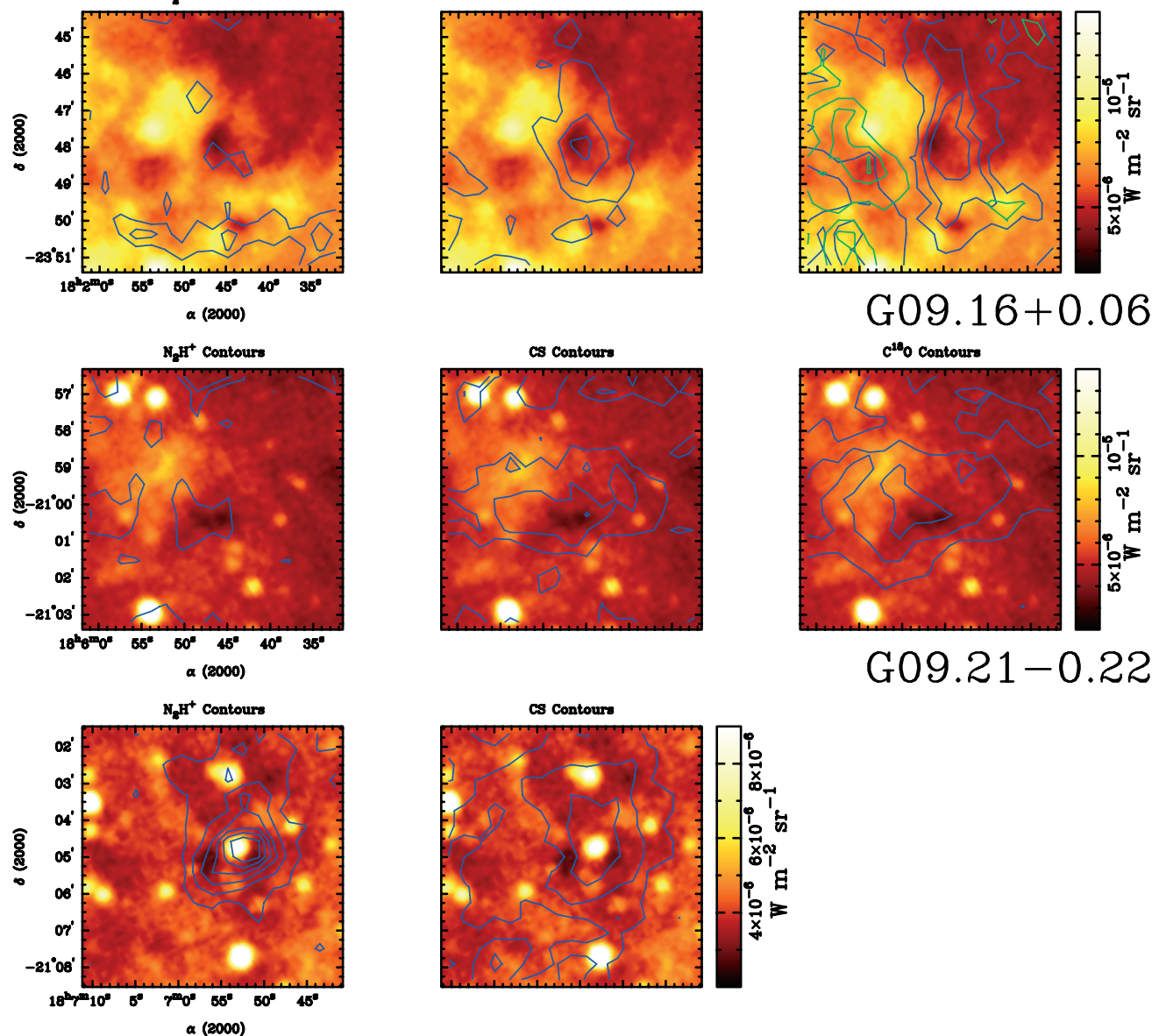

Fig. 1.-Color image shows the $8 \mu \mathrm{m} \mathrm{MSX}$, and the color bar indicates the emission intensity scale in $\mathrm{W} \mathrm{m}^{-2} \mathrm{sr}^{-1}$. The contours represent the molecular line integrated intensity, for which the levels are listed for each object. The integrated emission was calculated within a $6-10 \mathrm{~km} \mathrm{~s}^{-1}$ window for $\mathrm{C}^{18} \mathrm{O}$ and $\mathrm{CS}$. For $\mathrm{N}_{2} \mathrm{H}^{+}$ the window was $15-20 \mathrm{~km} \mathrm{~s}^{-1}$ with some gaps accounting for the clustering of the hyperfine components. $G 05.85-0.23$ : blue contours show emission at $17 \mathrm{~km} \mathrm{~s} \mathrm{~s}^{-1}$; green contours: $9 \mathrm{~km} \mathrm{~s}^{-1}$. Levels: $1,2 \mathrm{~K} \mathrm{~km} \mathrm{~s}^{-1}$ for all plots. G06.26-0.51: blue contours : $23 \mathrm{~km} \mathrm{~s}^{-1}$; green contours : $17 \mathrm{~km} \mathrm{~s}^{-1}$. Levels: $\mathrm{N}_{2} \mathrm{H}^{+}: 0.5,1 \mathrm{~K} \mathrm{~km} \mathrm{~s}^{-1}$. CS : $1,2,3,4 \mathrm{~K} \mathrm{~km} \mathrm{~s}^{-1} \cdot \mathrm{C}^{18} \mathrm{O}: 1,2,3 \mathrm{~K} \mathrm{~km} \mathrm{~s}^{-1}$ for both velocities. G09.16+0.06: blue contours : $31 \mathrm{~km} \mathrm{~s}^{-1}$. Levels: $\mathrm{N}_{2} \mathrm{H}^{+}: 1,2 \mathrm{~K} \mathrm{~km} \mathrm{~s}{ }^{-1}$. CS : $0.5,1,1.5 \mathrm{~K} \mathrm{~km} \mathrm{~s}{ }^{-1}$. $\mathrm{C}^{18} \mathrm{O}: 1,2,3 \mathrm{~K} \mathrm{~km} \mathrm{~s}^{-1}$. G09.21-0.22: blue contours : $43 \mathrm{~km} \mathrm{~s}^{-1}$. Levels: $\mathrm{N}_{2} \mathrm{H}^{+}: 2,4,6,8,10,12 \mathrm{~K} \mathrm{~km} \mathrm{~s}^{-1}$. CS: 1,2,3 K km s${ }^{-1}$. G09.28 $-0.15:$ blue contours : $42 \mathrm{~km}$ $\mathrm{s}^{-1}$. Levels: $\mathrm{N}_{2} \mathrm{H}^{+}: 1.5,3,4.5,6,7.5 \mathrm{~K} \mathrm{~km} \mathrm{~s}^{-1}$. CS: $0.5,1,1.5,2,3 \mathrm{~K} \mathrm{~km} \mathrm{~s}^{-1}$. G09.86-0.04: blue contours : $18 \mathrm{~km} \mathrm{~s}^{-1}$. Levels: $\mathrm{N}_{2} \mathrm{H}^{+}: 1.5,3,4.5,6,7.5 \mathrm{~K} \mathrm{~km} \mathrm{~s}{ }^{-1}$. CS: $0.5,1,1.5,2,3 \mathrm{~K} \mathrm{~km} \mathrm{~s}^{-1}$. G09.88 -0.11: blue contours : $17 \mathrm{~km} \mathrm{~s}^{-1}$. Levels: $\mathrm{N}_{2} \mathrm{H}^{+}: 1,2 \mathrm{~K} \mathrm{~km} \mathrm{~s}^{-1}$. CS: $0.5,1,1.5,2,3 \mathrm{~K} \mathrm{~km} \mathrm{~s}$. G10.59-0.31: blue contours : $17 \mathrm{~km}$ $\mathrm{s}^{-1}$. Levels: $3,5,7,9,11 \mathrm{~K} \mathrm{~km} \mathrm{~s}^{-1}$ for both molecules. G10.70-0.33: blue contours : $0 \mathrm{~km} \mathrm{~s}^{-1}$; green contours : $33 \mathrm{~km} \mathrm{~s}^{-1}$. Levels: $2,3,4,5 \mathrm{~K} \mathrm{~km} \mathrm{~s} \mathrm{~s}^{-1}$ for both molecules/velocities. G10.99-0.09: blue contours : $30 \mathrm{~km} \mathrm{~s}^{-1}$. Levels: $\mathrm{N}_{2} \mathrm{H}^{+}: 2,4,6,8,10 \mathrm{~K} \mathrm{~km} \mathrm{~s}^{-1}$. CS: $1,1.5,2 \mathrm{~K} \mathrm{~km} \mathrm{~s}^{-1}$. $\mathrm{C}^{18} \mathrm{O}: 2,3,4,5 \mathrm{~K} \mathrm{~km} \mathrm{~s}^{-1}$. G12.22+0.14: blue contours : $40 \mathrm{~km} \mathrm{~s}^{-1}$. Levels: $1,2,3,4,5 \mathrm{~K} \mathrm{~km} \mathrm{~s}^{-1}$ for each molecule. G12.50-0.22: blue contours : $36 \mathrm{~km} \mathrm{~s}^{-1}$. Levels: $\mathrm{N}_{2} \mathrm{H}^{+}: 2,4,6,8,10 \mathrm{~K} \mathrm{~km} \mathrm{~s}{ }^{-1}$. CS: $1,2,3,4 \mathrm{~K}$ $\mathrm{km} \mathrm{s}^{-1} \cdot \mathrm{C}^{18} \mathrm{O}: 1,2,3 \mathrm{~K} \mathrm{~km} \mathrm{~s}^{-1}$. G14.33-0.57: blue contours : $19 \mathrm{~km} \mathrm{~s}^{-1}$ (solid (a)), $20 \mathrm{~km} \mathrm{~s}^{-1}$ (dashed (b)) Levels: $\mathrm{N}_{2} \mathrm{H}^{+}: 2,4,6,8,10 \mathrm{~K} \mathrm{~km} \mathrm{~s}{ }^{-1}$. CS : 2,3,4,5,6,7 K $\mathrm{km} \mathrm{s}^{-1} \cdot \mathrm{C}^{18} \mathrm{O}: 4,5,6,7,8,9 \mathrm{~K} \mathrm{~km} \mathrm{~s}^{-1}$ (a); 2,3,4 $\mathrm{K} \mathrm{km} \mathrm{s}^{-1}$ (b). G19.37-0.03: blue contours : $27 \mathrm{~km} \mathrm{~s}^{-1}$. Levels: $\mathrm{N}_{2} \mathrm{H}^{+}: 1,3,5,7,9,11 \mathrm{~K} \mathrm{~km} \mathrm{~s}$. . CS : 1,2.5,4,5.5,7 K km $\mathrm{s}^{-1}$. G19.40-0.01: blue contours : $27 \mathrm{~km} \mathrm{~s}^{-1}$. Levels: $\mathrm{N}_{2} \mathrm{H}^{+}: 1,3,5,7,9,11 \mathrm{~K} \mathrm{~km} \mathrm{~s}^{-1}$. CS : 1,2.5,4,5.5,7 K km s${ }^{-1}$. G23.37-0.29: blue contours : 78 km s green contours : $103 \mathrm{~km} \mathrm{~s}^{-1}$; white contours : $65 \mathrm{~km} \mathrm{~s}^{-1}$. Levels: $\mathrm{N}_{2} \mathrm{H}^{+}: 3,6,9,12,15,18 \mathrm{~K} \mathrm{~km} \mathrm{~s}^{-1}\left(78 \mathrm{~km} \mathrm{~s}^{-1}\right.$ component); 4,6,8,10 K km s $3,4,5,6,7 \mathrm{~K} \mathrm{~km} \mathrm{~s}^{-1}\left(78 \mathrm{~km} \mathrm{~s}^{-1}\right) ; 3,4,5,6 \mathrm{~K} \mathrm{~km} \mathrm{~s}^{-1}\left(103 \mathrm{~km} \mathrm{~s}^{-1}\right) ; 3,4,5,6,9 \mathrm{~K} \mathrm{~km} \mathrm{~s}^{-1}\left(65 \mathrm{~km} \mathrm{~s}^{-1}\right)$. $\mathrm{C}^{18} \mathrm{O}: 5,7,9,11 \mathrm{~K} \mathrm{~km} \mathrm{~s}^{-1}\left(78 \mathrm{~km} \mathrm{~s}^{-1}\right) ; 3,6,9,12,15,18,21,24 \mathrm{~K} \mathrm{~km} \mathrm{~s}{ }^{-1}$ $\left(103 \mathrm{~km} \mathrm{~s}^{-1}\right) ; 3,6,9 \mathrm{~K} \mathrm{~km} \mathrm{~s}^{-1}\left(65 \mathrm{~km} \mathrm{~s}^{-1}\right)$. G23.48 -0.53: blue contours : $64 \mathrm{~km} \mathrm{~s}^{-1}$; green contours : $76 \mathrm{~km} \mathrm{~s}^{-1}$. Levels: $1,1.5,2,2.5 \mathrm{~K} k \mathrm{~km} \mathrm{~s}$ - both molecules/ velocities. G24.05-0.22: blue contours : $82 \mathrm{~km} \mathrm{~s}^{-1}$. Levels: $\mathrm{N}_{2} \mathrm{H}^{+}: 1,2,3 \mathrm{~K} \mathrm{~km} \mathrm{~s}^{-1}$. CS : 1,2,3,4 K km s${ }^{-1}$. $\mathrm{C}^{18} \mathrm{O}: 1,2,3,4,5 \mathrm{~K} \mathrm{~km} \mathrm{~s}$. G24.16+0.08: blue contours : $53 \mathrm{~km} \mathrm{~s}^{-1}$; green contours : $113 \mathrm{~km} \mathrm{~s}^{-1}$. Levels: $\mathrm{N}_{2} \mathrm{H}^{+}: 1,2,3 \mathrm{~K} \mathrm{~km} \mathrm{~s}^{-1}\left(113 \mathrm{~km} \mathrm{~s}^{-1}\right.$ component). CS : 1,2,3 K km s $\mathrm{km}^{-1}$ in both velocities. $\mathrm{C}^{18} \mathrm{O}: 1,2,3 \mathrm{~K} \mathrm{~km} \mathrm{~s} \mathrm{~K}^{-1}$ $\left(53 \mathrm{~km} \mathrm{~s}^{-1}\right) ; 2,4,6 \mathrm{~K} \mathrm{~km} \mathrm{~s}^{-1}\left(113 \mathrm{~km} \mathrm{~s}^{-1}\right)$. G25.99-0.06: blue contours : $90 \mathrm{~km} \mathrm{~s}^{-1}$. Levels: $\mathrm{N}_{2} \mathrm{H}^{+}: 1,2,3 \mathrm{~K} \mathrm{~km} \mathrm{~s}^{-1}$. CS : 1,2 K km s . G30.14-0.07: blue contours : 

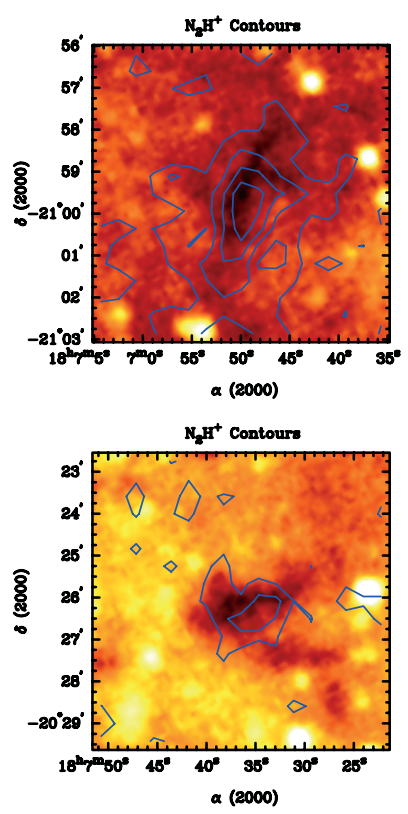

$\mathrm{N}_{8} \mathrm{H}^{+}$Contours
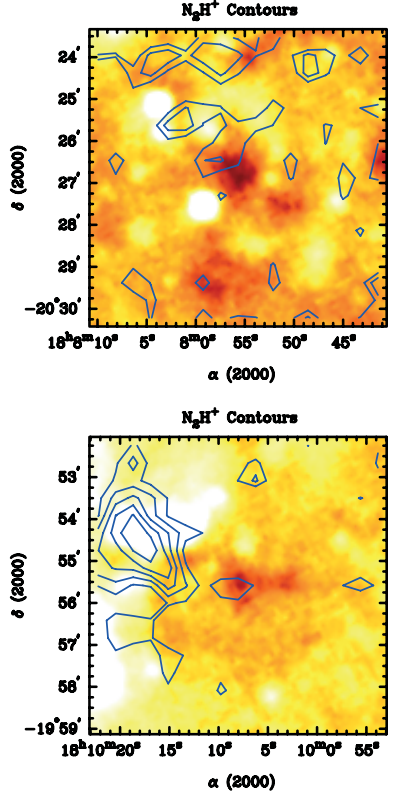

G09.28-0.15

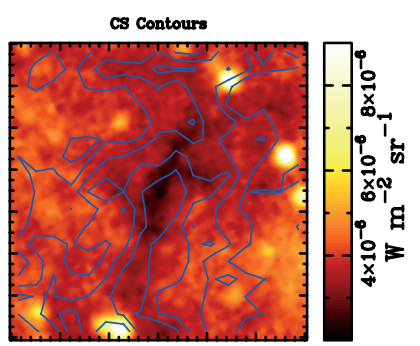

G09.86-0.04

cs Contours

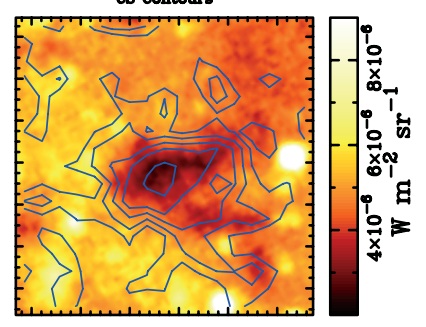

cs Contours
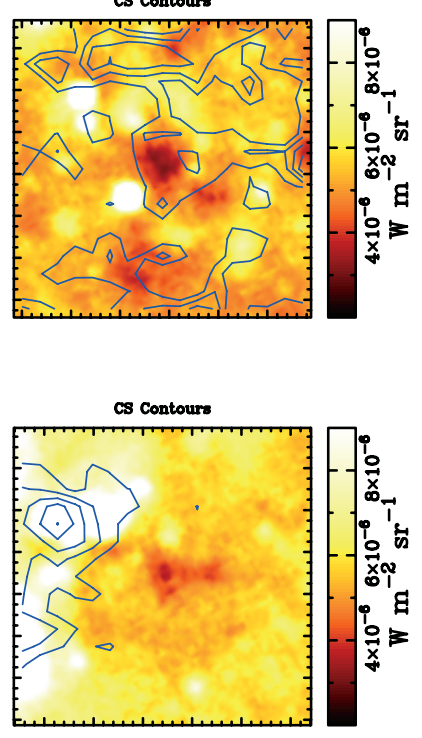

G09.88-0.11
G10.59-0.31

FIg. 1.-Continued. $87 \mathrm{~km} \mathrm{~s}^{-1}$. Levels: 0.5 for both molecules. G30.53-0.27: blue contours : $103 \mathrm{~km} \mathrm{~s}^{-1}$. Levels: $\mathrm{N}_{2} \mathrm{H}^{+}: 0.5 \mathrm{~K} \mathrm{~km} \mathrm{~s}{ }^{-1}$. CS : $1,1.5,2,2.5 \mathrm{~K}$ km $\mathrm{s}^{-1}$. G30.89+ 0.14: blue contours : $96 \mathrm{~km} \mathrm{~s}^{-1}$; green contours : $40 \mathrm{~km} \mathrm{~s}^{-1}$; white contours : $108 \mathrm{~km} \mathrm{~s}^{-1}$. Levels: $\mathrm{N}_{2} \mathrm{H}^{+}: 2,3,4 \mathrm{~K} \mathrm{~km} \mathrm{~s}{ }^{-1}$ (96 km s $\mathrm{km}^{-1}$ component); $2,4,6,8,10 \mathrm{~K} \mathrm{~km} \mathrm{~s}^{-1}\left(40 \mathrm{~km} \mathrm{~s}^{-1}\right) ; 2,4,6,8,10 \mathrm{~K} \mathrm{~km} \mathrm{~s}^{-1}\left(108 \mathrm{~km} \mathrm{~s}^{-1}\right)$. CS : $3,4,5,6,7 \mathrm{~K} \mathrm{~km} \mathrm{~s}^{-1}\left(96 \mathrm{~km} \mathrm{~s}^{-1}\right) ; 2,3,4 \mathrm{~K} \mathrm{~km} \mathrm{~s}^{-1}\left(40 \mathrm{~km} \mathrm{~s}^{-1}\right.$ and $108 \mathrm{~km} \mathrm{~s}^{-1}$ components). G30.98 -0.15: blue contours : $78 \mathrm{~km} \mathrm{~s}^{-1}$. Levels: $\mathrm{N}_{2} \mathrm{H}^{+}: 1,2,4,6,8 \mathrm{~K} \mathrm{~km} \mathrm{~s}^{-1}$. CS : 1,2,3,4 K km s${ }^{-1}$. G31.02-0.12: blue contours : $76 \mathrm{~km} \mathrm{~s}{ }^{-1}$; green contours : $83 \mathrm{~km} \mathrm{~s}^{-1}$; white contours : $92 \mathrm{~km} \mathrm{~s}^{-1}$. Levels: $\mathrm{N}_{2} \mathrm{H}^{+}: 1,2,3,4 \mathrm{~K} \mathrm{~km} \mathrm{~s}^{-1}\left(76 \mathrm{~km} \mathrm{~s}^{-1}\right.$ and $83 \mathrm{~km} \mathrm{~s}^{-1}$ components); 2,4,6,8,10 K km s${ }^{-1}\left(92 \mathrm{~km} \mathrm{~s}^{-1}\right)$. CS : 1,2,3,4,5,6,7 K $\mathrm{km} \mathrm{s}^{-1}\left(76 \mathrm{~km} \mathrm{~s}^{-1}\right) ; 1,2,3,4,5,6 \mathrm{~K} \mathrm{~km} \mathrm{~s}^{-1}\left(83 \mathrm{~km} \mathrm{~s}^{-1}\right) ; 1,2,3,4,5,6,9 \mathrm{~K} \mathrm{~km} \mathrm{~s}^{-1}\left(92 \mathrm{~km} \mathrm{~s}^{-1}\right)$. $\mathrm{C}^{18} \mathrm{O}: 1,2,3,4,5,6,7,8,9 \mathrm{~K} \mathrm{~km} \mathrm{~s}^{-1}\left(76 \mathrm{~km} \mathrm{~s}^{-1}\right) ; 1,2,3,6,9,12,15,18,21,24 \mathrm{~K}$ $\mathrm{km} \mathrm{s}^{-1}\left(83 \mathrm{~km} \mathrm{~s}^{-1}\right) ; 1,2,3 \mathrm{~K} \mathrm{~km} \mathrm{~s}^{-1}\left(92 \mathrm{~km} \mathrm{~s}^{-1}\right)$ G32.01+0.05: blue contours : $95 \mathrm{~km} \mathrm{~s}^{-1}$. Levels: $\mathrm{N}_{2} \mathrm{H}^{+}: 3,6,9,12,15,18,21,24 \mathrm{~K} \mathrm{~km} \mathrm{~s}$. . CS : 2,4,6,8,10 K km s${ }^{-1}$. $\mathrm{C}^{18} \mathrm{O}: 2,4,6,8,10 \mathrm{~K} \mathrm{~km} \mathrm{~s}^{-1}$. G33.82-0.22: blue contours : $11 \mathrm{~km} \mathrm{~s}^{-1}$. Levels: $\mathrm{N}_{2} \mathrm{H}^{+}: 1,2,3 \mathrm{~K} \mathrm{~km} \mathrm{~s}^{-1}$. CS : $0.5,1 \mathrm{~K} \mathrm{~km} \mathrm{~s}^{-1}$. G34.63-1.03: blue contours : $14 \mathrm{~km} \mathrm{~s}^{-1}$. Levels: $\mathrm{N}_{2} \mathrm{H}^{+}: 1,2,3 \mathrm{~K} \mathrm{~km} \mathrm{~s}^{-1}$. CS : $0.5,1.0 \mathrm{~K} \mathrm{~km} \mathrm{~s}^{-1}$. $\mathrm{C}^{18} \mathrm{O}: 0.5,1,1.5 \mathrm{~K} \mathrm{~km} \mathrm{~s}^{-1}$. G34.74-0.12: blue contours : $79 \mathrm{~km} \mathrm{~s}^{-1}$. Levels: $\mathrm{N}_{2} \mathrm{H}^{+}: 1,2,3 \mathrm{~K} \mathrm{~km} \mathrm{~s}{ }^{-1}$. CS : $1,2,3,4 \mathrm{~K} \mathrm{~km} \mathrm{~s}^{-1}$. $\mathrm{C}^{18} \mathrm{O}: 1,2,3,4,5 \mathrm{~K} \mathrm{~km} \mathrm{~s}^{-1}$. G34.78 -0.80: blue contours : $44 \mathrm{~km} \mathrm{~s}^{-1}$; green contours : $37 \mathrm{~km} \mathrm{~s}^{-1}$. Levels: $\mathrm{N}_{2} \mathrm{H}^{+}: 1,2,3,4 \mathrm{~K} \mathrm{~km} \mathrm{~s}^{-1}$ for both velocities. CS : $1,2,3 \mathrm{~K} \mathrm{~km} \mathrm{~s}^{-1}$ for both velocities. $\mathrm{C}^{18} \mathrm{O}: 1,2,3 \mathrm{~K} \mathrm{~km} \mathrm{~s}^{-1}$ (44 $\mathrm{km} \mathrm{s}^{-1}$ component); $1,2,3,4,5,6 \mathrm{~K} \mathrm{~km} \mathrm{~s}^{-1}\left(37 \mathrm{~km} \mathrm{~s}^{-1}\right)$. G35.20-0.72: blue contours : $33 \mathrm{~km} \mathrm{~s}{ }^{-1}$. Levels: $\mathrm{N}_{2} \mathrm{H}^{+}: 2,4,6,8,10,12,14 \mathrm{~K} \mathrm{~km} \mathrm{~s}^{-1}$. CS : 2,4,6,8,10,12 $\mathrm{K} \mathrm{km} \mathrm{s}^{-1}$. $\mathrm{C}^{18} \mathrm{O}: 2,4,6,8,10,12,14 \mathrm{~K} \mathrm{~km} \mathrm{~s}^{-1}$. G37.44+0.14: blue contours : $40 \mathrm{~km} \mathrm{~s}{ }^{-1}$; green contours : $18 \mathrm{~km} \mathrm{~s}^{-1}$; white contours : $86 \mathrm{~km} \mathrm{~s}^{-1}$. Levels: $0.5,1,2,3 \mathrm{~K} \mathrm{~km} \mathrm{~s}^{-1}$ for all molecules/velocities. G37.89-0.15: blue contours : $13 \mathrm{~km} \mathrm{~s}^{-1}$; green contours: $65 \mathrm{~km} \mathrm{~s}^{-1}$; white contours : $86 \mathrm{~km} \mathrm{~s}^{-1}$. Levels: $\mathrm{N}_{2} \mathrm{H}^{+}: 0.5 \cdot 1,2,3 \mathrm{~K} \mathrm{~km} \mathrm{~s}^{-1}\left(13 \mathrm{~km} \mathrm{~s}^{-1}\right.$ component). $\mathrm{CS}: 0.5 \cdot 1,2,3 \mathrm{~K} \mathrm{~km} \mathrm{~s}^{-1}\left(13 \mathrm{~km} \mathrm{~s}^{-1}\right) . \mathrm{C}^{18} \mathrm{O}: 0.5,1,2,3 \mathrm{~K} \mathrm{~km} \mathrm{~s}^{-1}\left(13 \mathrm{~km} \mathrm{~s}^{-1} \mathrm{and}^{2} \mathrm{~km} \mathrm{~s}^{-1}\right) ; 2,3 \mathrm{~K}$ $\mathrm{km} \mathrm{s}^{-1}\left(86 \mathrm{~km} \mathrm{~s}^{-1}\right)$. G50.07+0.06: blue contours $55 \mathrm{~km} \mathrm{~s}^{-1}$. Levels: $0.5,1 \mathrm{~K} \mathrm{~km} \mathrm{~s}^{-1}$ for both molecules. 
G10.70-0.33
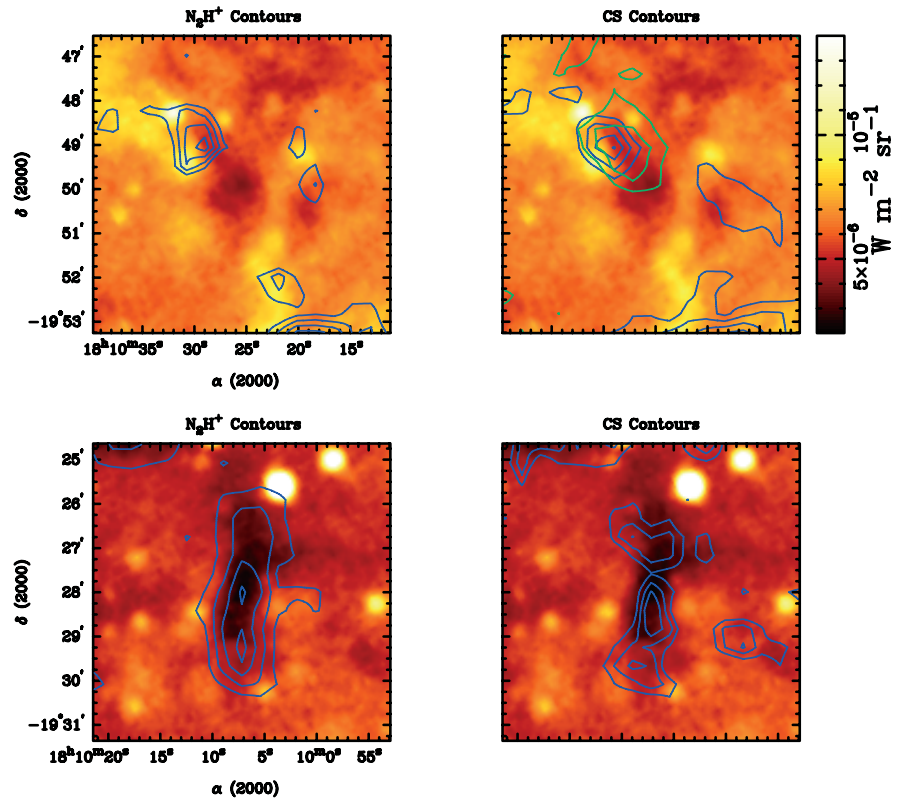

G10.99-0.09

$c^{180}$ contours

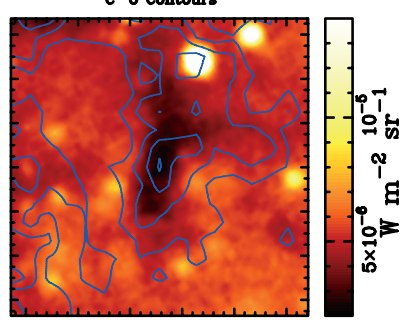

G12.22+0.14

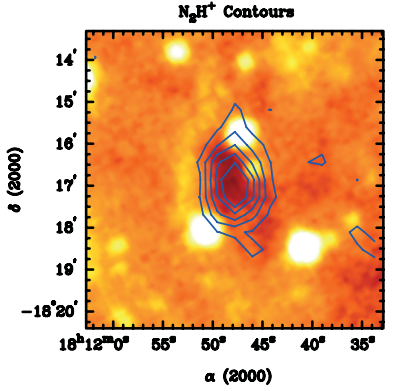

cs Contours

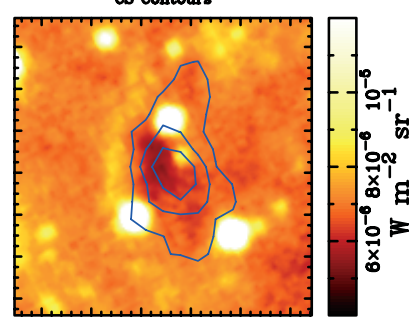

G12.50-0.22
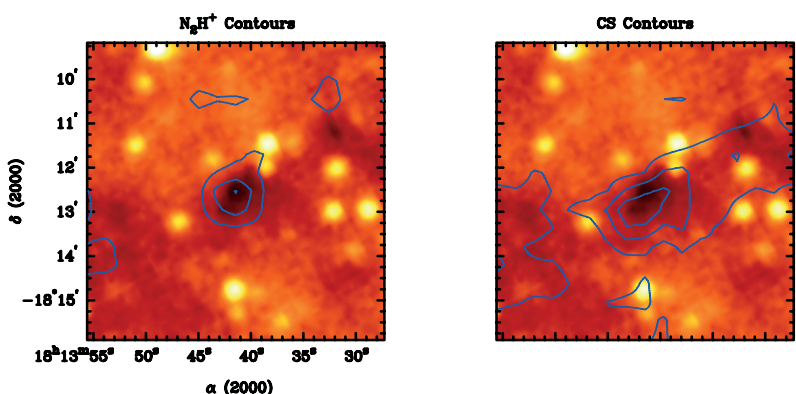

$\mathrm{C}^{18} \mathrm{O}$ Contours

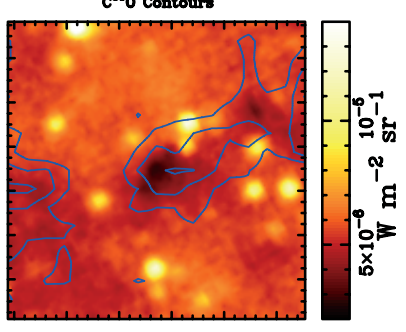

FIG. 1._Continued 

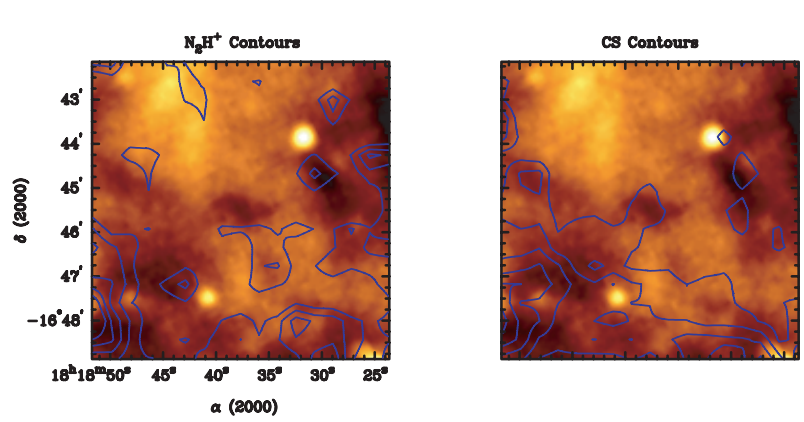

G14.33-0.57
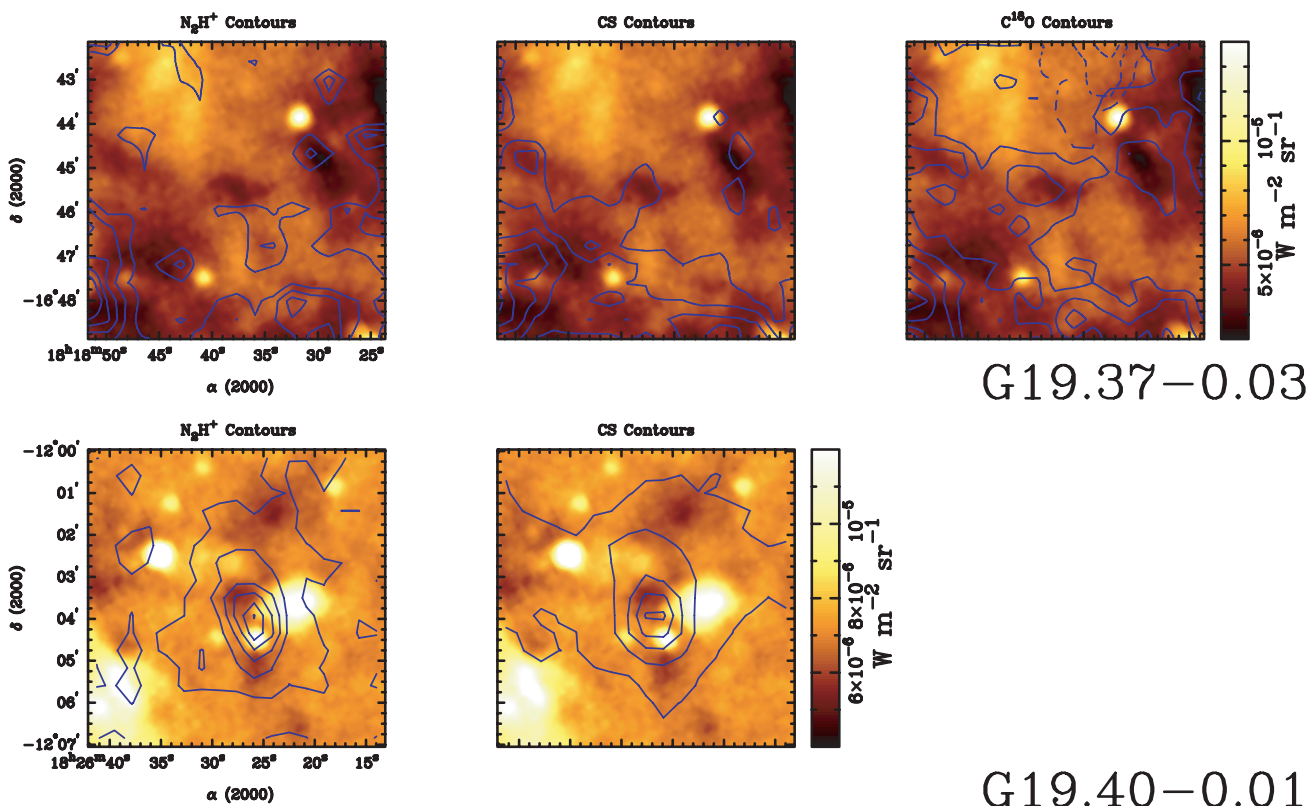

G19.37-0.03
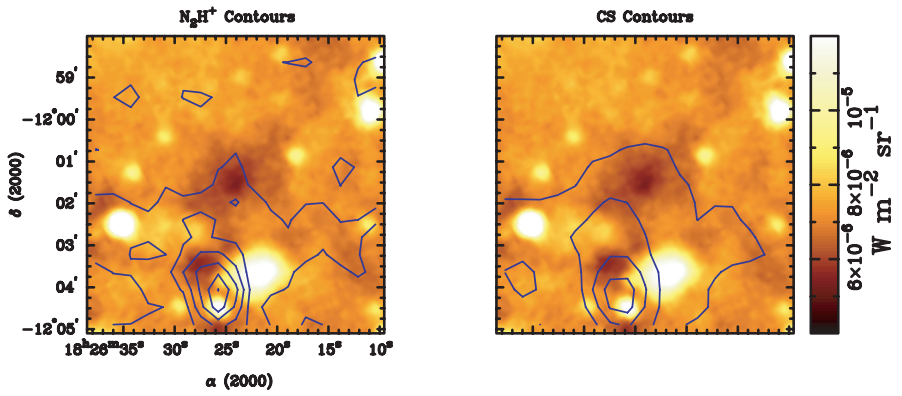

G19.40-0.01
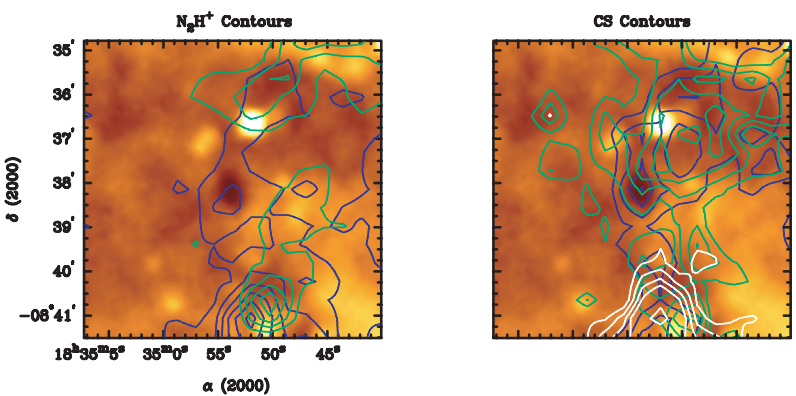

G23.37-0.29

$\mathrm{c}^{18} \mathrm{O}$ Contours

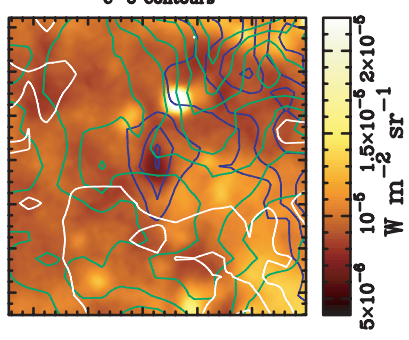

FIG. 1.-Continued 

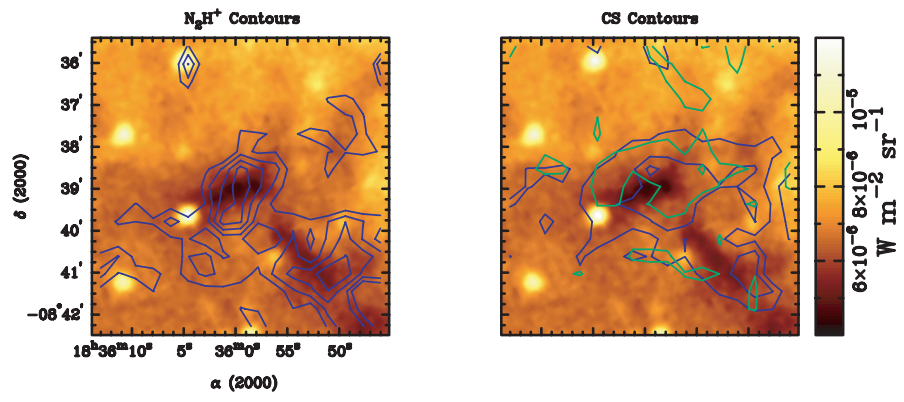

G23.48-0.53
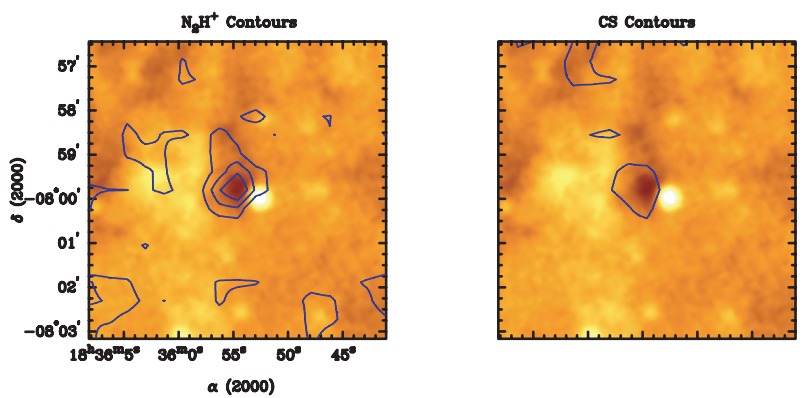

G24.05-0.22
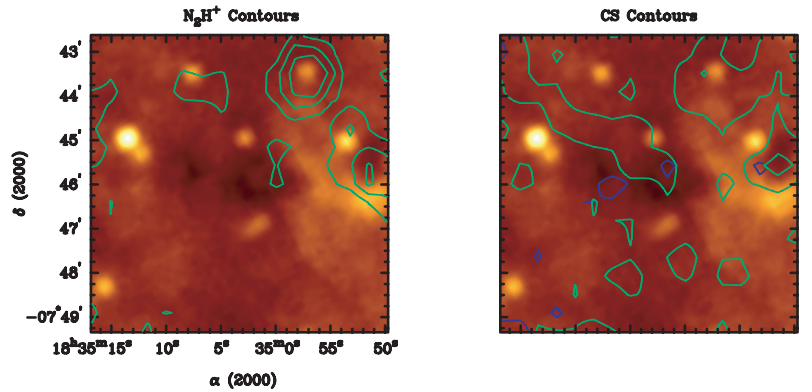

$\mathrm{c}^{180} \mathrm{O}$ contours

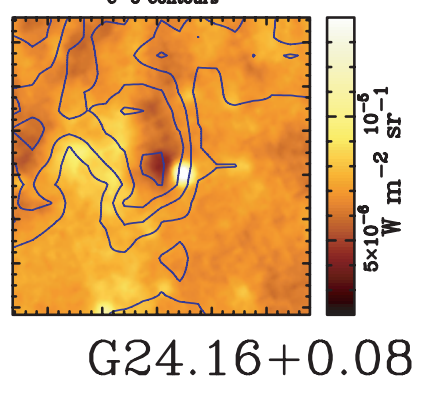

$\mathrm{C}^{100} \mathrm{O}$ contours
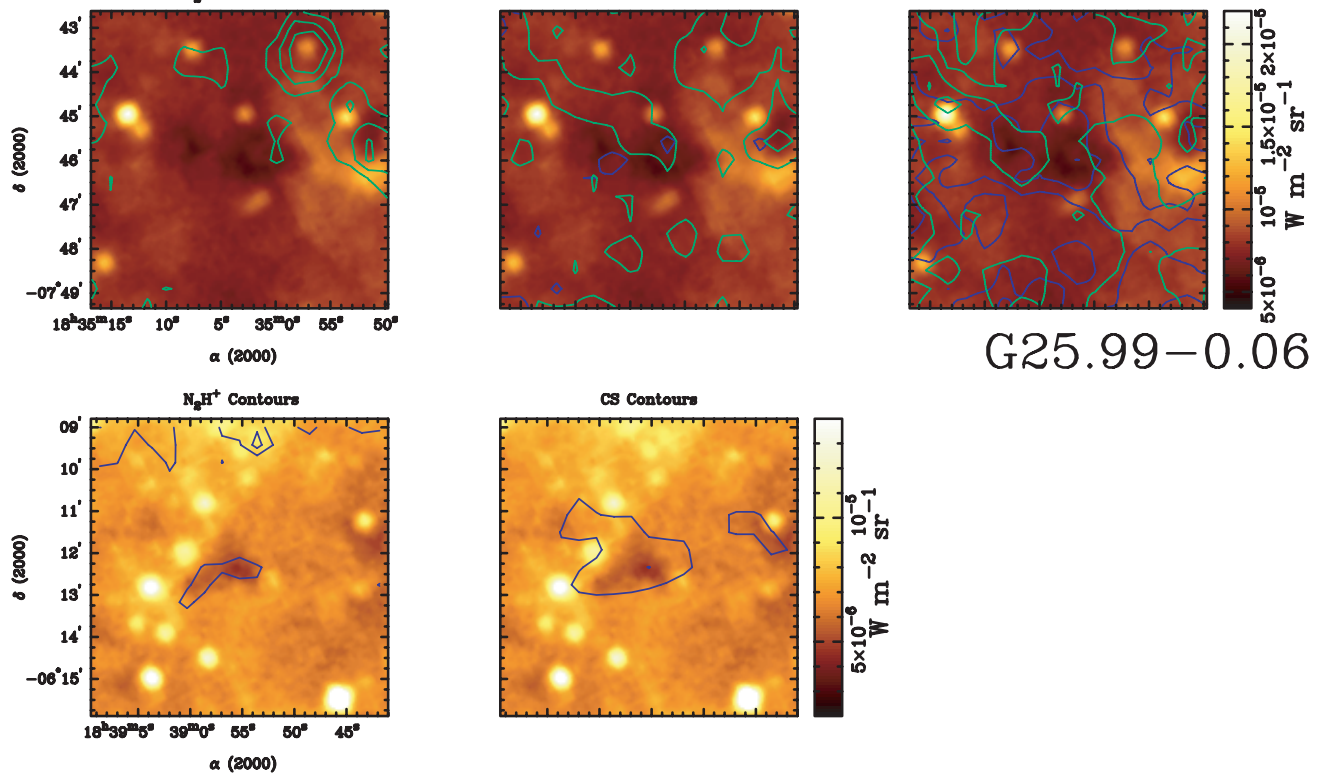

FIG. 1._Continued 

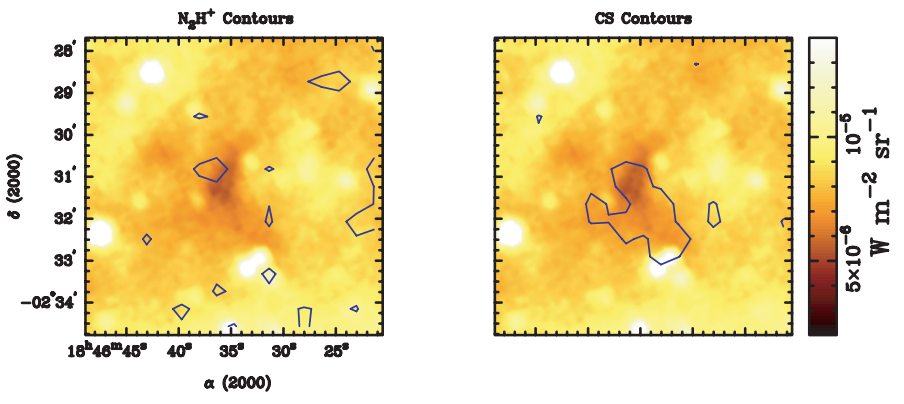

G30.14-0.07
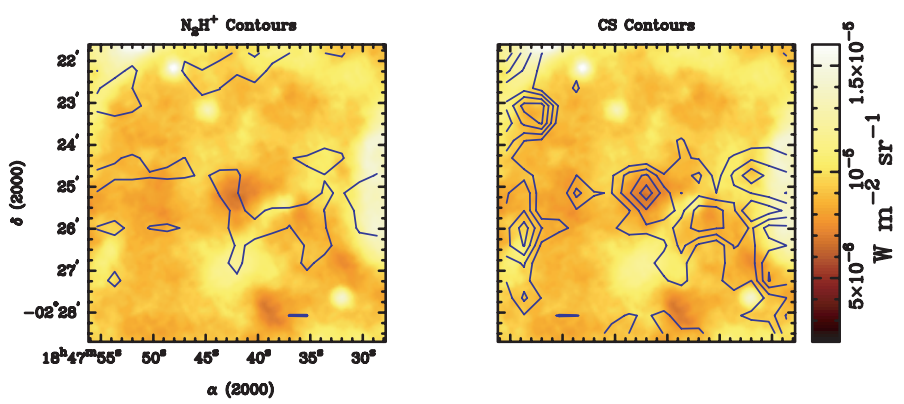

G30.53-0.27
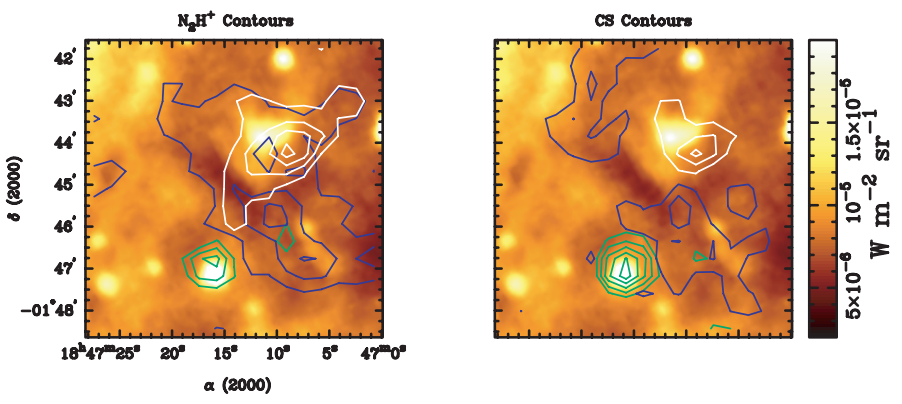

G30.89+0.14
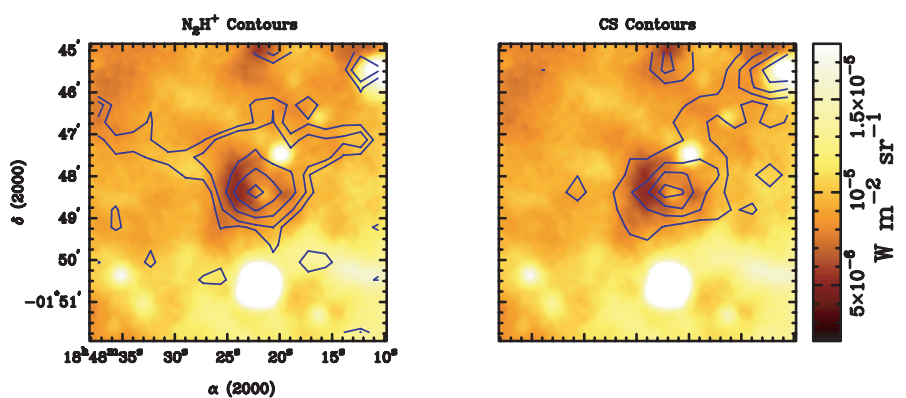

G30.98-0.15

FIg. 1.-Continued 

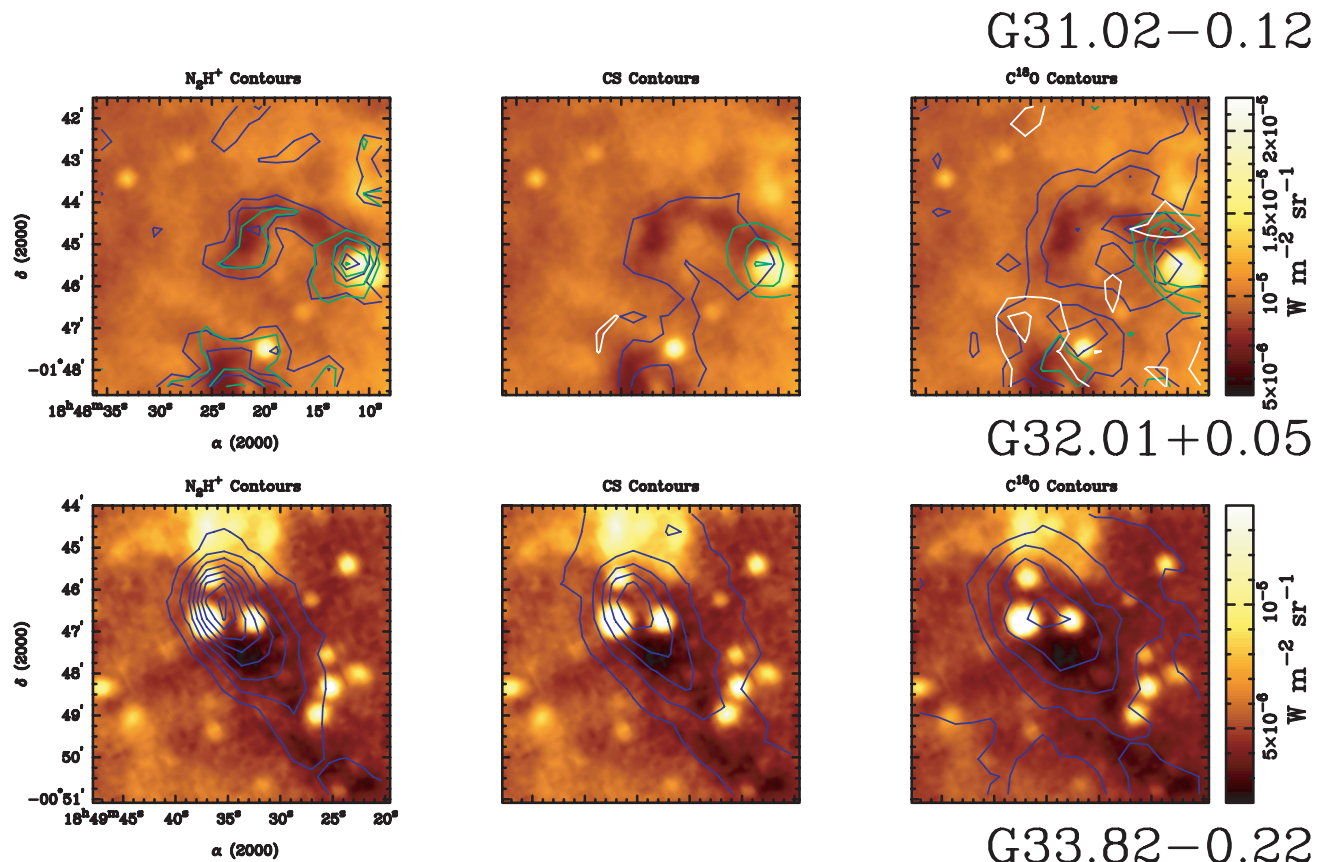

G33.82-0.22
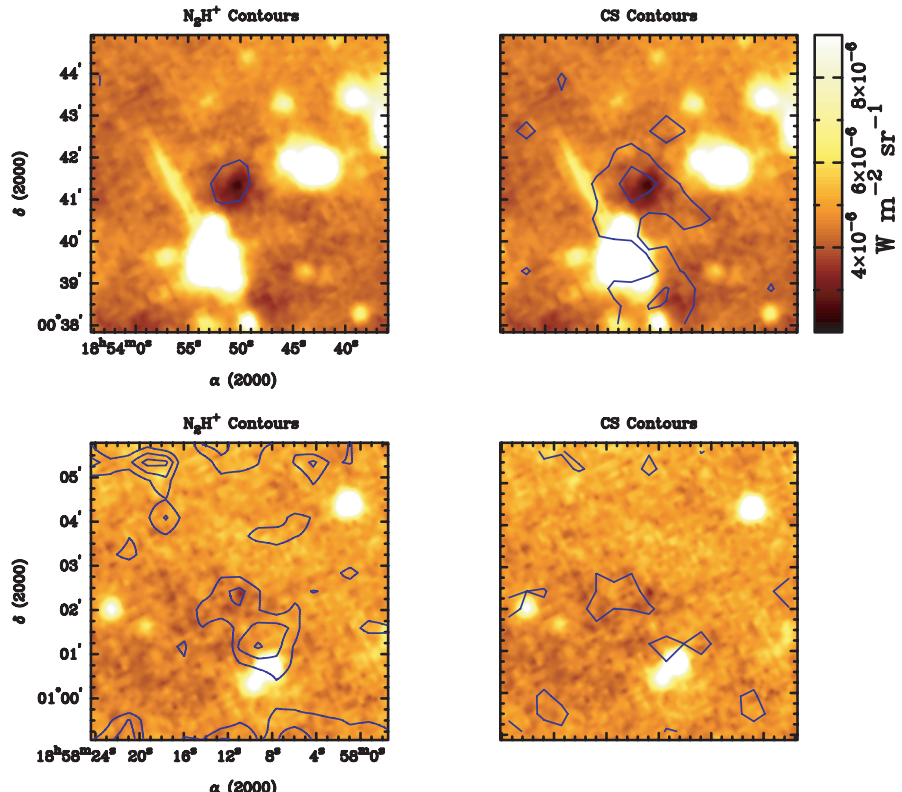

G34.63-1.03

$\mathrm{c}^{180} \mathrm{O}$ contours

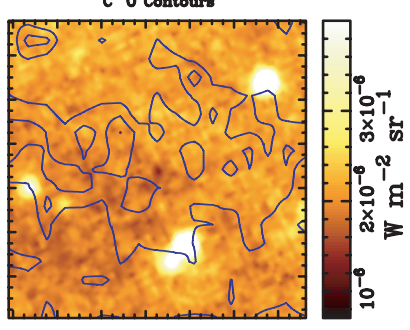

FIg. 1.-Continued 

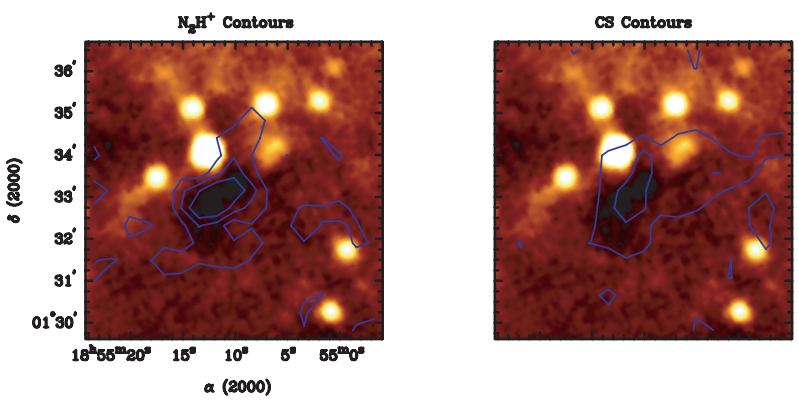

G34.74-0.12
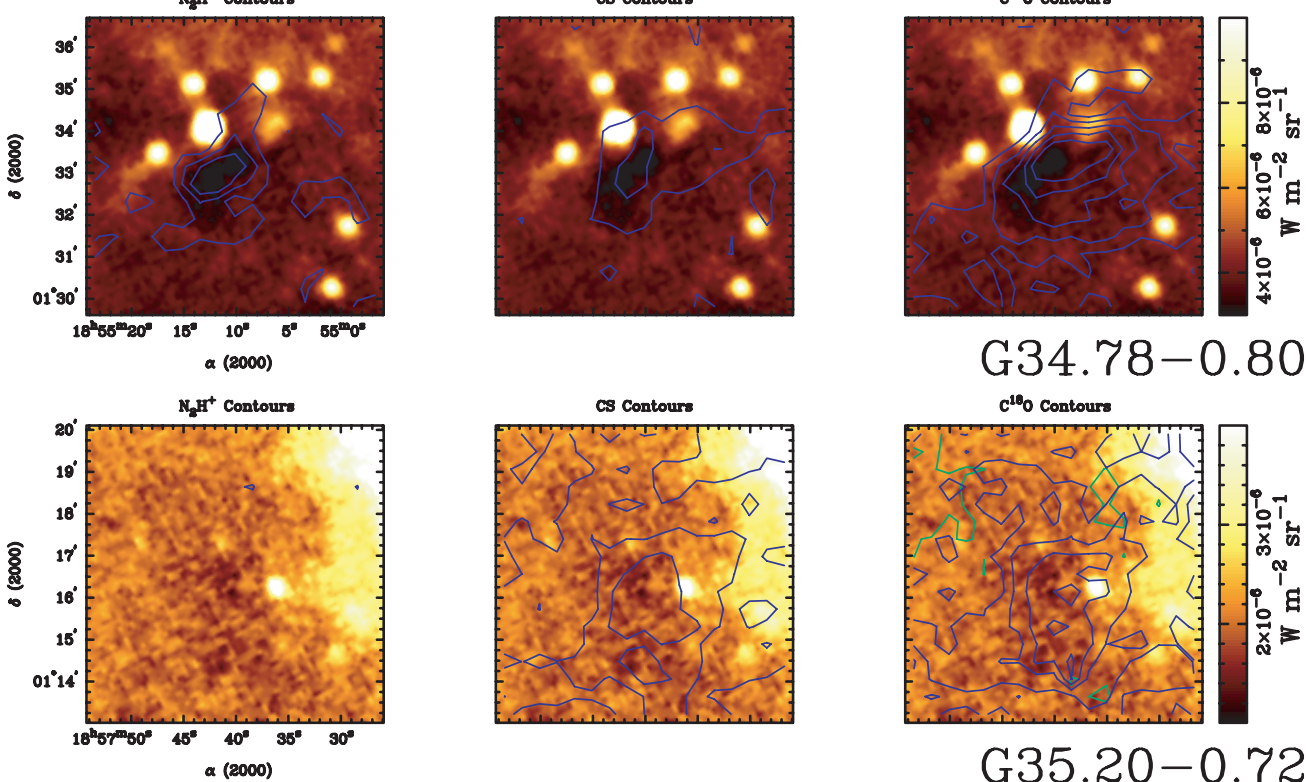

$\mathrm{N}_{8} \mathrm{H}^{+}$Contours
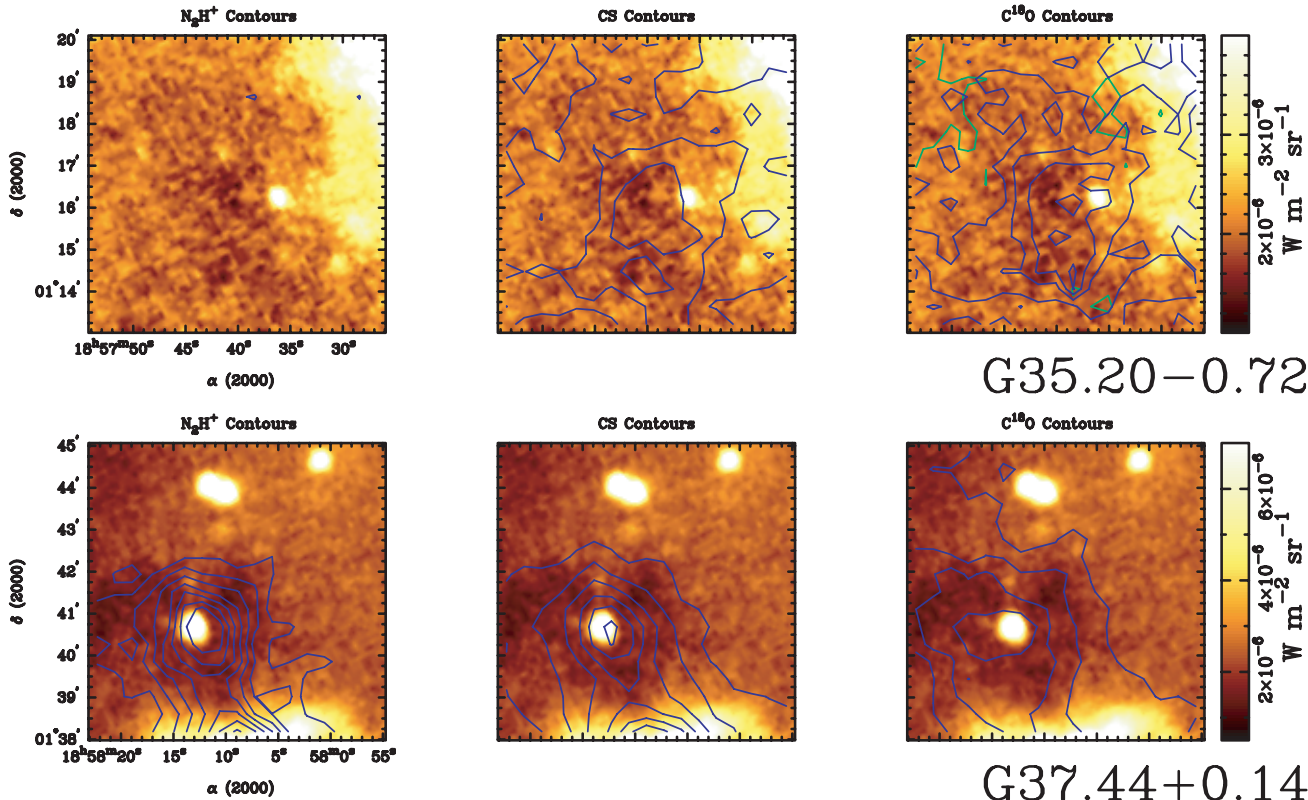

G35.20-0.72

$c^{10} 0$ contours

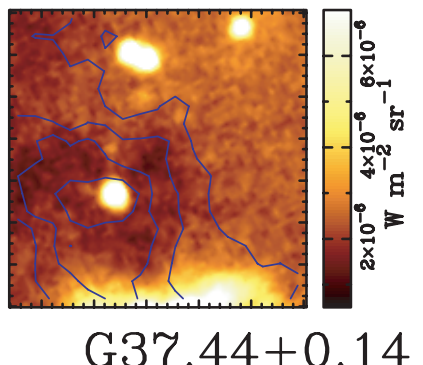

$\mathrm{c}^{10} \mathrm{o}$ Contours
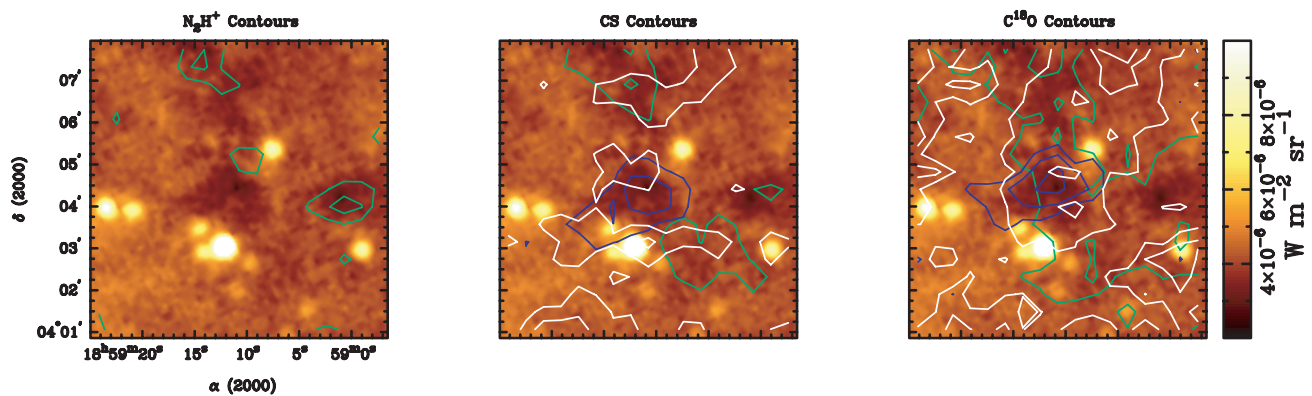

FIG. 1.-Continued 

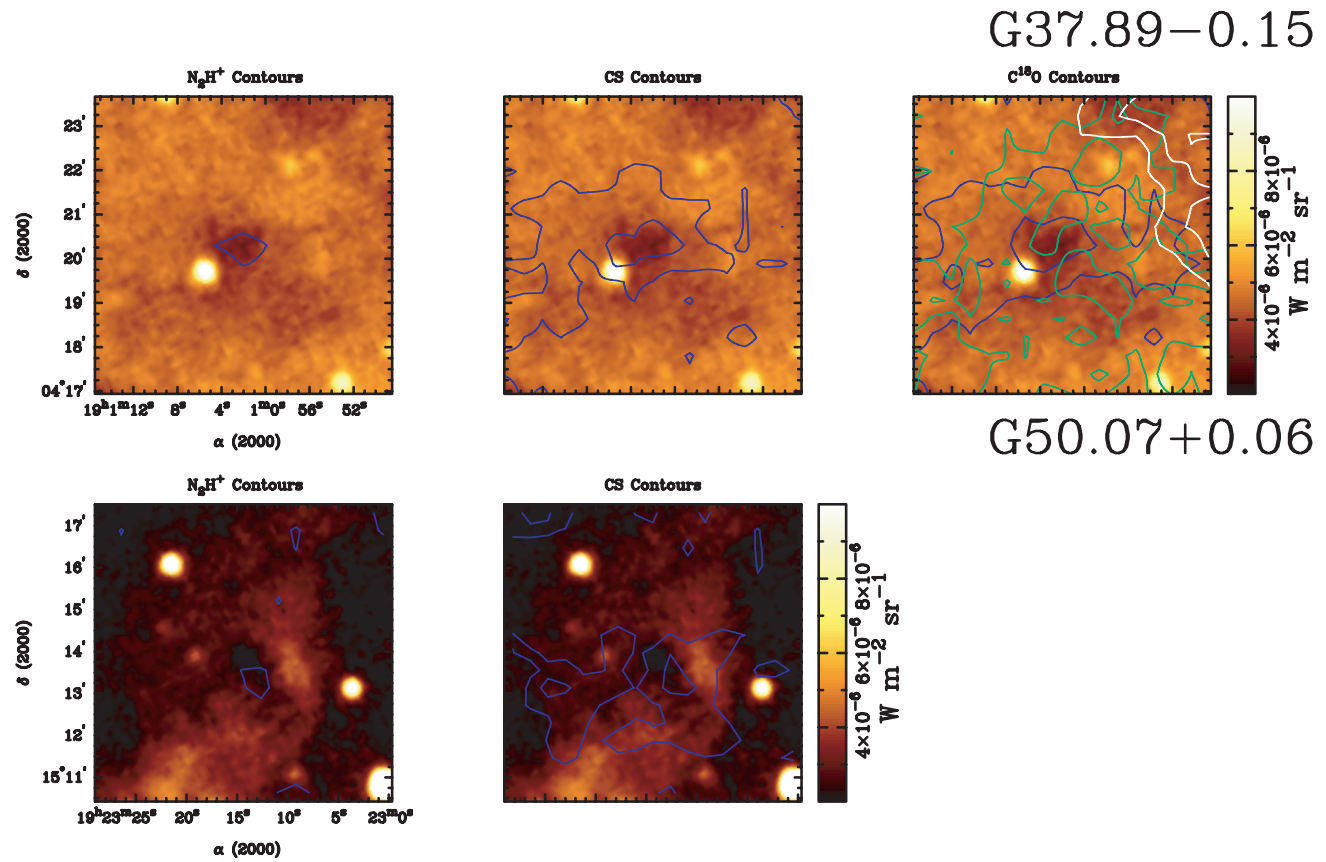

Fig. 1.-Continued

to the UC H II region $(12.0 \mathrm{kpc})$. In this case, it is likely that we are detecting two clouds along the same line of sight at different distances: one near the UC H II region and one nearer to us. Since the emission of the primary component corresponds so well to the absorbing dark cloud, we maintain that these lie at the "near" distances, although there is significant uncertainty in the distance calculation. Nonetheless, as we will show, these clouds are massive and are likely associated with the formation of intermediateand high-mass stars and stellar clusters.

\subsection{Column Densities and Densities}

To determine the molecular abundances relative to molecular hydrogen, we also need a measure of the total $\mathrm{H}_{2}$ column density. We estimate $N\left(\mathrm{H}_{2}\right)$ from the $M S X$ images convolved to match the FCRAO beam resolution and the simple relation $\tau_{\lambda}=$ $\sigma_{\lambda} \cdot N\left(\mathrm{H}_{2}\right)$, where $\tau_{\lambda}$ is the dust opacity, $\sigma_{\lambda}$ is the dust extinction cross section, and $N\left(\mathrm{H}_{2}\right)$ is the column density of molecular hydrogen. The behavior of the mid-infrared extinction law is an area of active research. Recent results from Indebetouw et al. (2005) show agreement with Weingartner \& Draine (2001) $\left(R_{v}=\right.$ 5.5 , "case B"), and we therefore adopt a value for $\sigma$, at $8.8 \mu \mathrm{m}$ of $2.3 \times 10^{-23} \mathrm{~cm}^{2}$, although this value can be considered reliable only within a factor of 2 . The opacity, $\tau_{\lambda}$, is roughly estimated by examining the relative intensities of the average background $\left(I_{o, \lambda}\right)$ and central core $\left(I_{\lambda}\right)$, assuming that $I_{\lambda}=I_{o, \lambda} e^{-\tau_{\lambda}}$ (i.e., no emission by the core itself). The ratio $I_{\lambda} / I_{o, \lambda}$ is related, but not identical, to the brightness contrast listed in Table 1, since the values listed in Table 1 do not incorporate the convolution to the FCRAO beam, and are provided at the original $M S X$ resolution.

The column densities of $\mathrm{C}^{18} \mathrm{O}$ and $\mathrm{CS}$ are estimated by assuming that the cores are in local thermodynamic equilibrium (LTE) at a temperature of $15 \mathrm{~K}$ and that the emission is optically thin (Table 4). While the optically thin assumption is probably reasonable for $\mathrm{C}^{18} \mathrm{O}$, it probably does not hold for CS, which generally has optically thick emission in the interstellar medium. For $\mathrm{N}_{2} \mathrm{H}^{+}$, the fits to the hyperfine components generally suggest low optical depth, or $\tau \sim 1$. However, its emission is likely not in
LTE. At a density of $10^{5} \mathrm{~cm}^{-3}$, the fractional population will be underestimated by a factor of $\sim 1.7$ relative to LTE; we therefore apply this correction factor. We also crudely estimate the gas density by assuming the cloud is spherical and dividing the $\mathrm{H}_{2}$ column density by the diameter of the cloud (using the sizes listed in Table 1 and the distances in Table 3 ). This gives an average density of $\approx 5000 \mathrm{~cm}^{-3}$. This is well below the average densities found in other studies of regions of massive star formation using other tracers. For example, Plume et al. (1997) surveyed multiple transitions of CS in $150 \mathrm{H}_{2} \mathrm{O}$ masers (used as signposts of massive star formation) and found an average gas density of $7.9 \times 10^{5} \mathrm{~cm}^{-3}$. An obvious explanation for the lower density in our sample is that the clouds are not spherical, as we have naively assumed, and may instead be clumpy on scales below our resolution. Moreover, these objects are likely at an earlier evolutionary state, which is characterized by lower densities. A more detailed analysis of the density and column density will be presented in future work (D. L. Gibson et al. 2007, in preparation).

\subsection{Masses}

The total mass of each dark cloud is estimated using an assumed ("near") distance, an approximate size based on the extent of molecular emission, the molecular column densities, and approximate abundance calculated at the peak of absorption. Table 4 lists the masses of the objects for which there is a significant detection of $\mathrm{N}_{2} \mathrm{H}^{+}$or $\mathrm{C}^{18} \mathrm{O}$ (or both) and a distance could be assigned. Since some of the dark clouds shown in Figure 1 have a different structure when viewed in different molecular tracers and may have different opacities, we have, for completeness, estimated the masses independently based on the abundances derived from both the $\mathrm{N}_{2} \mathrm{H}^{+}$and $\mathrm{C}^{18} \mathrm{O}$ data. Our average mass is $\approx 2000-3000 M_{\odot}$ (depending on whether the $\mathrm{N}_{2} \mathrm{H}^{+}$or $\mathrm{C}^{18} \mathrm{O}$ mass is used).

Figure 2 shows how the masses of our sample compare to the high-mass protostellar objects (HMPOs) presented in Williams et al. (2004), which were determined from submillimeter continuum emission. The masses for the HMPO sample are shown 
TABLE 3

Kinematic Distances to Separated Velocity Components

\begin{tabular}{|c|c|c|c|c|c|}
\hline Source & $\begin{array}{l}\text { Velocity } \\
\text { Component } \\
\left(\mathrm{km} \mathrm{s}^{-1}\right)\end{array}$ & $\begin{array}{c}\text { Near } \\
\text { Distance } \\
(\mathrm{kpc})\end{array}$ & $\begin{array}{c}\text { Far } \\
\text { Distance } \\
(\mathrm{kpc})\end{array}$ & $\begin{array}{c}\text { Adopted } \\
\text { Distance } \\
(\mathrm{kpc})\end{array}$ & $\begin{array}{c}\text { UC H II Region } \\
\text { Distance } \\
(\mathrm{kpc})\end{array}$ \\
\hline G05.85-0.23........ & $\begin{array}{r}17 \\
9\end{array}$ & $\begin{array}{l}3.14_{-0.76}^{+0.66} \\
1.53_{-0.96}^{+0.86}\end{array}$ & $\begin{array}{l}13.78_{-0.67}^{+0.75} \\
15.38_{-0.86}^{+0.97}\end{array}$ & 3.14 & 2.6 \\
\hline G06.26-0.51......... & $\begin{array}{l}23 \\
17\end{array}$ & $\begin{array}{l}3.78_{-0.67}^{-0.59} \\
3.01_{-0.77}^{+0.68}\end{array}$ & $\begin{array}{l}13.12_{-0.60}^{+0.67} \\
13.89_{-0.68}^{+0.77}\end{array}$ & 3.78 & 2.6 \\
\hline G09.16+0.06 ......... & 31 & $3.81_{-0.69}^{+0.61}$ & $12.97_{-0.61}^{+0.69}$ & 3.81 & 6.2 \\
\hline G09.21-0.22 ...... & 43 & $4.57_{-0.59}^{+0.53}$ & $12.21_{-0.53}^{+0.59}$ & 4.57 & 6.2 \\
\hline 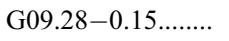 & 42 & $4.48_{-0.61}^{+0.54}$ & $12.30_{-0.54}^{+0.61}$ & 4.48 & 6.2 \\
\hline G09.86-0.04........ & 18 & $2.36_{-0.88}^{+0.78}$ & $14.39_{-0.78}^{+0.87}$ & 2.36 & 6.0 \\
\hline G10.99-0.09....... & 30 & $3.31_{-0.76}^{+0.69}$ & $13.37_{-0.68}^{+0.77}$ & 3.31 & 6.0 \\
\hline G12.22+0.14 ........ & 40 & $3.75_{-0.72}^{+0.65}$ & $12.86_{-0.65}^{+0.73}$ & 3.75 & 5.2 \\
\hline G12.50-0.22....... & 36 & $3.55_{-0.75}^{+0.67}$ & $13.05_{-0.67}^{+0.75}$ & 3.55 & 5.2 \\
\hline G14.33-0.57........ & $\begin{array}{l}19 \\
20\end{array}$ & $\begin{array}{l}1.99_{-0.95}^{+0.85} \\
2.04_{-0.94}^{+0.85}\end{array}$ & $\begin{array}{l}14.48_{-0.85}^{+0.95} \\
14.43_{-0.85}^{+0.94}\end{array}$ & $\begin{array}{l}1.99 \\
2.04\end{array}$ & 2.1 \\
\hline G19.37-0.03 $\ldots \ldots \ldots$ & 27 & $2.26_{-0.98}^{+0.84}$ & $13.78_{-0.88}^{+0.97}$ & 2.26 & 4.5 \\
\hline G19.40-0.01....... & 27 & $2.23_{-0.98}^{+0.88}$ & $13.81_{-0.89}^{+0.90}$ & 2.23 & 4.5 \\
\hline $\mathrm{G} 23.37-0.29 \ldots \ldots \ldots$ & $\begin{array}{r}78 \\
103 \\
65\end{array}$ & $\begin{array}{l}4.70_{-0.88}^{+0.90} \\
5.69_{-0.91}^{+1.20} \\
4.13_{-0.89}^{+0.88}\end{array}$ & $\begin{array}{r}10.91_{-0.91}^{+0.88} \\
9.91_{-1.20}^{+0.92} \\
11.47_{-0.87}^{+0.90}\end{array}$ & 4.70 & 9.0 \\
\hline G23.48-0.53......... & $\begin{array}{l}64 \\
76\end{array}$ & $\begin{array}{l}4.10_{-0.90}^{+0.88} \\
4.60_{-0.88}^{+0.91}\end{array}$ & $\begin{array}{c}11.50_{-0.89}^{+0.89} \\
10.99_{-0.91}^{+0.88}\end{array}$ & 4.10 & 9.0 \\
\hline $\mathrm{G} 24.05-0.22 \ldots \ldots \ldots$ & 82 & $4.82_{-0.90}^{-0.98}$ & $10.70_{-0.96}^{+0.91}$ & 4.82 & 9.0 \\
\hline $\mathrm{G} 24.16+0.08 \ldots \ldots \ldots$ & $\begin{array}{r}53 \\
113\end{array}$ & $\begin{array}{l}3.46_{-0.94}^{+0.91} \\
6.13_{-1.03} .03\end{array}$ & $\begin{array}{c}12.05_{-0.91}^{+0.94} \\
9.38\end{array}$ & 3.46 & 9.0 \\
\hline 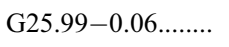 & 90 & $5.15_{-0.99}^{+1.23}$ & $10.13_{-1.23}^{+0.99}$ & 5.15 & 14.0 \\
\hline G30.89+0.14 ........ & $\begin{array}{r}96 \\
40 \\
108\end{array}$ & $\begin{array}{l}5.65_{-1.38} \\
2.62_{-1.14}^{+1.14} \\
6.65_{-1.84}\end{array}$ & $\begin{array}{c}8.93 \\
11.97_{-1.14}^{+1.15} \\
7.94\end{array}$ & 5.65 & 8.5 \\
\hline G30.98-0.15 ........ & 78 & $4.63_{-1.19}^{+1.63}$ & $9.94_{-1.62}^{+1.19}$ & 4.63 & 8.5 \\
\hline $\mathrm{G} 31.02-0.12 \ldots \ldots \ldots$ & $\begin{array}{l}76 \\
83 \\
92\end{array}$ & $\begin{array}{l}4.56_{-1.19}^{+1.56} \\
4.90_{-1.23} \\
5.41_{-1.33}\end{array}$ & $\begin{array}{c}10.01_{-1.56}^{+1.19} \\
9.67 \\
9.16\end{array}$ & 4.56 & 8.5 \\
\hline $\mathrm{G} 32.01+0.05 \ldots \ldots \ldots$ & 95 & $5.77_{-1.51}$ & 8.64 & 5.77 & 8.5 \\
\hline $\mathrm{G} 34.63-1.03 \ldots \ldots \ldots$ & 14 & $0.84^{+1.26}$ & $13.14_{-1.26}^{+1.32}$ & 3.2 & \\
\hline G34.74-0.12....... & 79 & $4.86_{-1.45}$ & 9.11 & 4.86 & 3.7 \\
\hline G34.78-0.80........ & $\begin{array}{l}44 \\
37\end{array}$ & $\begin{array}{l}2.80_{-1.26}^{+1.33} \\
2.41_{-1.26}^{+1.28}\end{array}$ & $\begin{array}{l}11.17_{-1.33}^{+1.25} \\
11.56_{-1.29}^{+1.26}\end{array}$ & 2.80 & 3.2 \\
\hline G35.20-0.72 ....... & 33 & $2.17_{-1.28}^{+1.20}$ & $11.73_{-1.30}^{+1.27}$ & 2.17 & 3.2 \\
\hline G37.44+0.14 ....... & $\begin{array}{l}40 \\
18 \\
86\end{array}$ & $\begin{array}{l}2.59_{-1.34}^{+1.47} \\
1.16^{+1.34} \\
5.90_{-2.12}\end{array}$ & $\begin{array}{c}10.91_{-1.47}^{+1.34} \\
12.34_{-1.34}^{+1.36} \\
7.60\end{array}$ & 2.59 & 12.0 \\
\hline G37.89-0.15........ & $\begin{array}{l}13 \\
65 \\
86\end{array}$ & $\begin{array}{l}0.82^{+1.35} \\
4.16_{-1.51} \\
6.09_{-2.31}\end{array}$ & $\begin{array}{l}12.60_{-1.35}^{+1.38} \\
9.26 \\
7.33\end{array}$ & 0.82 & 12.0 \\
\hline
\end{tabular}

for both their "near" and "far" kinematic distances. Assuming that the sample encompasses objects at both the "near" and "far" kinematic distances, then the derived masses for HMPOs show a comparable range to our sources.

One caveat with our comparison to the Williams et al. sample lies in the selection bias of our sample. As we will discuss below, our observations are only sensitive to relatively massive objects when the cores reside at such large distances. Furthermore, the HMPOs in the Williams et al. study also contain protostars that heat the surrounding environment and increase the dust emission. Therefore, in the warmer environments of the Williams et al. survey, lower mass cores would be easier to detect. The mass distribution shown in Figure 2 also shows good agreement with that found by Shirley et al. (2003), who observed CS emission from a sample of massive star-forming regions and found a mean mass of $920 M_{\odot}$ with a large dispersion.
Several assumptions contribute to the uncertainty in the mass calculation, which is dominated by the error in the abundance calculation. We assume a constant temperature of $15 \mathrm{~K}$, and a $5 \mathrm{~K}$ change in this value results in a $\sim 20 \%$ change in the abundance. The uncertainty in the dust opacity/column density relation contributes another factor of 2. Finally, we assume a constant abundance along the line of sight, which likely contributes an additional factor of 2-3 to the mass estimates.

We note that the typical distance to these clouds is $\sim 4 \mathrm{kpc}$, and with $50^{\prime \prime}$ resolution, we are likely only sensitive to objects of some minimum mass. To examine this limit, we modeled the emission of a cloud assuming a constant density of $10^{5} \mathrm{~cm}^{-3}$, a radius of $0.1 \mathrm{pc}$, and an $\mathrm{N}_{2} \mathrm{H}^{+}$abundance of $5 \times 10^{-10}$ using a Monte Carlo radiation transfer model (Ashby et al. 2000). We estimate that our observations are capable of detecting clouds of mass greater than $50-100 M_{\odot}$ at a distance of $4 \mathrm{kpc}$ with a $50^{\prime \prime}$ beam. 
TABLE 4

Molecular Abundances and Cloud Masses

\begin{tabular}{|c|c|c|c|c|c|c|c|}
\hline \multirow[b]{2}{*}{ SOURCE } & \multirow{2}{*}{$\begin{array}{c}\text { Distance }^{\mathrm{a}} \\
(\mathrm{kpc})\end{array}$} & \multirow{2}{*}{$\begin{array}{c}N\left(\mathrm{H}_{2}\right) \\
\left(10^{21} \mathrm{~cm}^{-2}\right)\end{array}$} & \multicolumn{3}{|c|}{ Abundance Relative to $\mathrm{H}_{2}$} & \multicolumn{2}{|c|}{ Cloud Mass } \\
\hline & & & $\mathrm{N}_{2} \mathrm{H}^{+}$ & $\mathrm{C}^{18} \mathrm{O}$ & $\mathrm{CS}$ & $\left(M_{\odot}\right)^{\mathrm{b}}$ & $\left(M_{\odot}\right)^{\mathrm{c}}$ \\
\hline G05.85-0.23 ............. & 3.14 & $4.4 \pm 0.9$ & $2.9 \mathrm{E}-10$ & $3.5 \mathrm{E}-7$ & $5.0 \mathrm{E}-10$ & $2.6 \mathrm{E}+2$ & $2.5 \mathrm{E}+2$ \\
\hline G06.26-0.51_............. & 3.78 & $13.5 \pm 1.2$ & $\ldots$ & $1.3 \mathrm{E}-7$ & $5.6 \mathrm{E}-10$ & $\ldots$ & $6.2 \mathrm{E}+3$ \\
\hline G09.16+0.06 ................ & 3.81 & $4.4 \pm 1.6$ & $\ldots$ & $3.4 \mathrm{E}-7$ & $4.2 \mathrm{E}-10$ & $\ldots$ & $3.3 \mathrm{E}+3$ \\
\hline G09.21-0.22 .............. & 4.57 & $1.9 \pm 1.2$ & $2.0 \mathrm{E}-9$ & $\ldots$ & $3.2 \mathrm{E}-9$ & $1.4 \mathrm{E}+3$ & $\ldots$ \\
\hline G09.28-0.15 .............. & 4.48 & $7.5 \pm 1.2$ & $5.2 \mathrm{E}-10$ & $\ldots$ & $7.9 \mathrm{E}-10$ & $3.4 \mathrm{E}+3$ & $\ldots$ \\
\hline G09.86-0.04 ............... & 2.36 & $9.8 \pm 1.0$ & $9.4 \mathrm{E}-11$ & $\ldots$ & $7.2 \mathrm{E}-10$ & $1.5 \mathrm{E}+3$ & \\
\hline G10.99-0.09 .............. & 3.32 & $8.1 \pm 0.9$ & $5.5 \mathrm{E}-10$ & $3.0 \mathrm{E}-7$ & $5.7 \mathrm{E}-10$ & $2.0 \mathrm{E}+3$ & $4.3 \mathrm{E}+3$ \\
\hline G12.22+0.14 .............. & 3.75 & $2.8 \pm 1.0$ & $1.4 \mathrm{E}-9$ & $\ldots$ & $3.0 \mathrm{E}-9$ & $3.2 \mathrm{E}+2$ & $\ldots$ \\
\hline 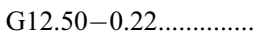 & 3.55 & $4.8 \pm 1.7$ & $6.5 \mathrm{E}-10$ & $3.6 \mathrm{E}-7$ & $1.4 \mathrm{E}-9$ & $5.4 \mathrm{E}+2$ & $7.1 \mathrm{E}+3$ \\
\hline G14.33-0.57a .............. & 1.99 & $4.1 \pm 0.4$ & & $5.7 \mathrm{E}-7$ & $1.4 \mathrm{E}-9$ & $\ldots$ & $8.3 \mathrm{E}+2$ \\
\hline G14.33-0.57b.............. & 2.05 & $3.4 \pm 1.1$ & $4.4 \mathrm{E}-10$ & $5.7 \mathrm{E}-7$ & $1.1 \mathrm{E}-9$ & $1.3 \mathrm{E}+3$ & $1.2 \mathrm{E}+3$ \\
\hline G19.37-0.03 ............. & 2.26 & $2.2 \pm 1.5$ & $1.7 \mathrm{E}-9$ & $\ldots$ & $6.4 \mathrm{E}-9$ & $2.8 \mathrm{E}+2$ & $\ldots$ \\
\hline G19.40-0.01_............. & 2.23 & $4.9 \pm 1.0$ & $2.1 \mathrm{E}-10$ & $\ldots$ & $7.7 \mathrm{E}-10$ & $2.1 \mathrm{E}+3$ & $\ldots$ \\
\hline $\mathrm{G} 23.37-0.20 \ldots \ldots \ldots \ldots$ & 4.70 & $3.4 \pm 1.1$ & $9.0 \mathrm{E}-10$ & $1.2 \mathrm{E}-6$ & $3.1 \mathrm{E}-9$ & $3.3 \mathrm{E}+3$ & $4.1 \mathrm{E}+3$ \\
\hline $\mathrm{G} 23.48-0.53 \mathrm{a} \ldots \ldots \ldots \ldots$ & 4.10 & $7.8 \pm 3.3$ & $2.0 \mathrm{E}-10$ & $\ldots$ & $5.4 \mathrm{E}-10$ & $2.7 \mathrm{E}+3$ & $\ldots$ \\
\hline $\mathrm{G} 23.48-0.53 \mathrm{~b} \ldots \ldots \ldots \ldots$ & 4.02 & $5.7 \pm 1.4$ & $2.3 \mathrm{E}-10$ & $\ldots$ & $6.0 \mathrm{E}-10$ & $2.0 \mathrm{E}+3$ & $\ldots$ \\
\hline G24.05-0.22 ............ & 4.82 & $2.7 \pm 1.4$ & $1.1 \mathrm{E}-9$ & $9.1 \mathrm{E}-7$ & $1.7 \mathrm{E}-9$ & $4.0 \mathrm{E}+2$ & $2.1 \mathrm{E}+3$ \\
\hline G24.16+0.08 ….......... & 3.46 & $4.0 \pm 1.4$ & $\ldots$ & $4.4 \mathrm{E}-7$ & $\ldots$ & $\ldots$ & $2.6 \mathrm{E}+3$ \\
\hline G25.99-0.06 .............. & 5.15 & $4.1 \pm 1.5$ & $2.1 \mathrm{E}-10$ & $\ldots$ & $1.2 \mathrm{E}-9$ & $6.8 \mathrm{E}+2$ & $\ldots$ \\
\hline G30.89+0.14 ….......... & 5.65 & $4.2 \pm 1.0$ & $3.2 \mathrm{E}-10$ & $\ldots$ & $6.2 \mathrm{E}-10$ & $1.1 \mathrm{E}+4$ & $\ldots$ \\
\hline 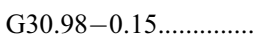 & 4.63 & $7.3 \pm 2.7$ & $6.6 \mathrm{E}-10$ & $\ldots$ & $1.4 \mathrm{E}-9$ & $1.9 \mathrm{E}+3$ & $\ldots$ \\
\hline G31.02-0.12 $\ldots \ldots \ldots \ldots$ & 4.56 & $4.3 \pm 0.9$ & $3.9 \mathrm{E}-10$ & $3.1 \mathrm{E}-7$ & $7.1 \mathrm{E}-10$ & $2.6 \mathrm{E}+3$ & $4.7 \mathrm{E}+3$ \\
\hline G32.01+0.05 .............. & 5.77 & $7.3 \pm 2.3$ & $1.0 \mathrm{E}-9$ & $6.5 \mathrm{E}-7$ & $2.6 \mathrm{E}-9$ & $8.7 \mathrm{E}+3$ & $1.3 \mathrm{E}+4$ \\
\hline G34.63-1.03 .............. & 0.84 & $3.6 \pm 1.0$ & $3.4 \mathrm{E}-10$ & $1.7 \mathrm{E}-7$ & $\ldots$ & $5.5 \mathrm{E}+1$ & $6.0 \mathrm{E}+1$ \\
\hline 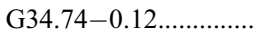 & 4.86 & $5.1 \pm 1.5$ & $4.0 \mathrm{E}-10$ & $7.3 \mathrm{E}-7$ & $1.0 \mathrm{E}-9$ & $8.7 \mathrm{E}+2$ & $2.4 \mathrm{E}+3$ \\
\hline $\mathrm{G} 34.78-0.80 \ldots \ldots \ldots \ldots$ & 2.80 & $6.1 \pm 3.8$ & $5.7 \mathrm{E}-11$ & $3.9 \mathrm{E}-7$ & $1.4 \mathrm{E}-9$ & 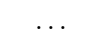 & $2.9 \mathrm{E}+3$ \\
\hline G35.20-0.72 $\ldots \ldots \ldots \ldots \ldots$ & 2.17 & $3.5 \pm 2.5$ & $9.2 \mathrm{E}-10$ & $6.2 \mathrm{E}-7$ & $2.5 \mathrm{E}-9$ & $1.0 \mathrm{E}+3$ & $1.5 \mathrm{E}+3$ \\
\hline $\mathrm{G} 37.44+0.14 \mathrm{a} \ldots \ldots \ldots \ldots$ & 2.59 & $3.0 \pm 1.5$ & $\ldots$ & $4.0 \mathrm{E}-7$ & $1.2 \mathrm{E}-9$ & $\ldots$ & $8.9 \mathrm{E}+1$ \\
\hline G37.44+0.14b ............ & 1.16 & $2.6 \pm 0.9$ & $2.6 \mathrm{E}-10$ & $2.1 \mathrm{E}-7$ & $4.5 \mathrm{E}-10$ & $3.2 \mathrm{E}+1$ & $1.1 \mathrm{E}+2$ \\
\hline G37.89-0.15 ............ & 0.82 & $3.2 \pm 1.6$ & $1.5 \mathrm{E}-10$ & $2.1 \mathrm{E}-7$ & $5.2 \mathrm{E}-10$ & $3.7 \mathrm{E}+0$ & $5.4 \mathrm{E}+1$ \\
\hline
\end{tabular}

${ }^{\text {a }}$ Distances from rotation curve of Fich et al. (1989), assuming absorbing cloud lies at near distance.

b From $\mathrm{N}_{2} \mathrm{H}^{+}$

${ }^{c}$ From $\mathrm{C}^{18} \mathrm{O}$.

Assuming that the "near" distance assumption is correct, the uncertainties mentioned above can account for up to a factor of 4-6 in mass error, as the 14\% error in translating the Galactic rotation curve to kinematic distances only introduces a distance error of $\sim 20 \%$. This accounting suggests that these objects are

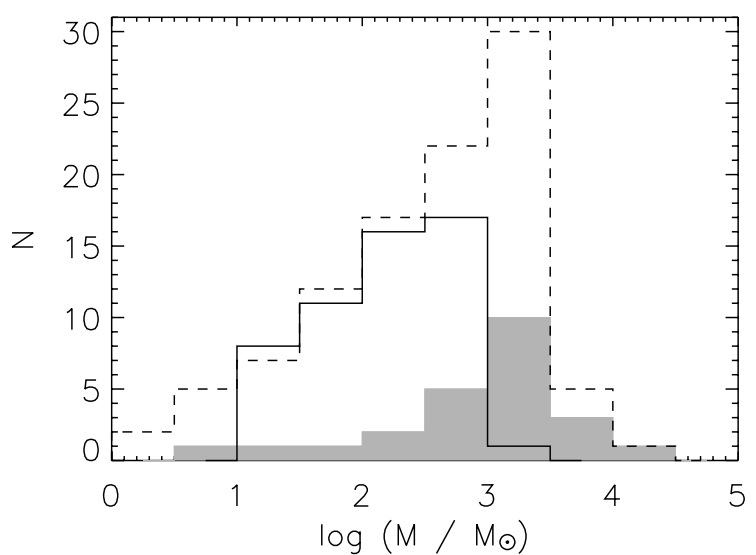

at least $100 M_{\odot}$, and likely an order of magnitude more massive. Should the "near" distance assumption be incorrect and the dark clouds lie closer to the "far" kinematic distance, then the distance error dominates the calculation, and these clouds are substantially more massive.

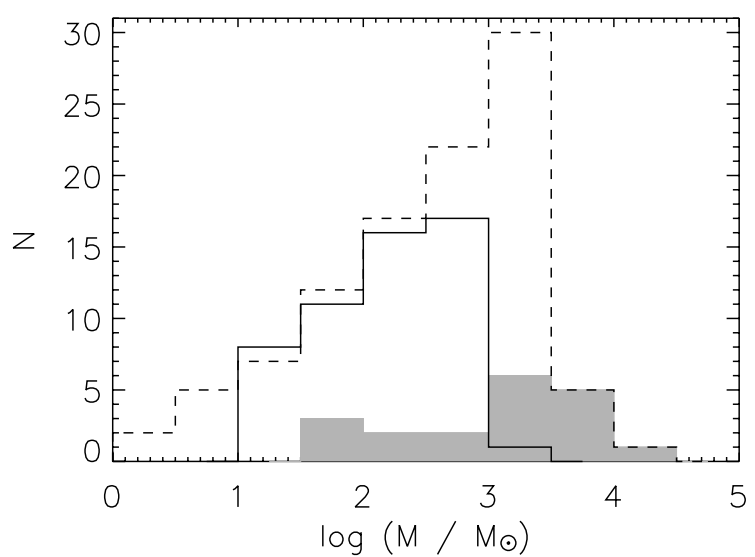

Fig. 2. - Histogram of the mass distribution for our sample of IR dark cores as derived from the $\mathrm{N}_{2} \mathrm{H}^{+}$data (left panel) and the $\mathrm{C}^{18} \mathrm{O}$ data (right panel). The gray histograms show the mass distribution for our sample of cores, whereas the solid line shows the distribution of masses of the Williams et al. (2004) cores (derived from $850 \mu \mathrm{m}$ data) based on the near Galactic distances. The dashed line represents the masses based on the far Galactic distances. 


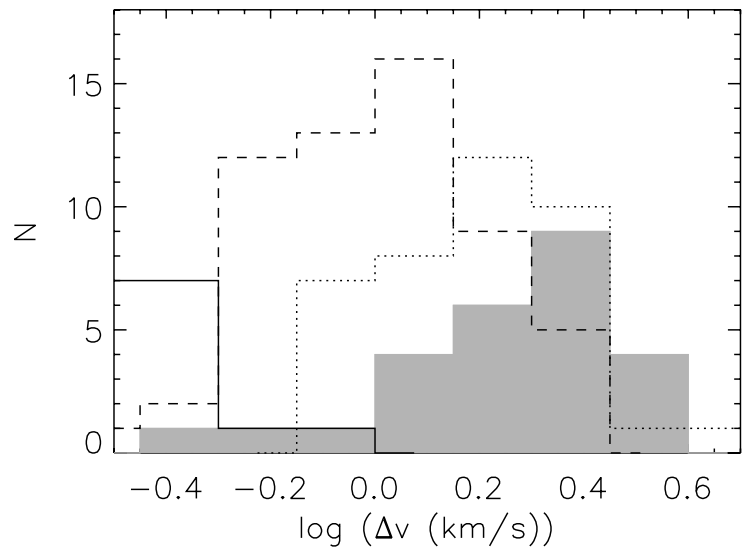

FIG. 3.- Histogram of the $\mathrm{N}_{2} \mathrm{H}^{+}$(solid line, Caselli et al. 2002; gray, this sample) and $\mathrm{NH}_{3}$ (dashed line; Harju et al. 1993; dotted line; Molinari et al. 1996) line widths.

\subsection{Velocity Dispersion}

The width of emission lines in star-forming clouds serves as a useful diagnostic in determining the nature of a molecular region. According to Goldsmith (1987), the sites of massive star formation, GMCs, are characterized by large line widths, while the isolated sites of low-mass star formation, dark clouds, have considerably smaller line widths. We illustrate this range in Figure 3.

Caselli et al. (2002) derived line widths for $\mathrm{N}_{2} \mathrm{H}^{+}$for a sample of low-mass, dense clumps in dark clouds, a site in which we expect to find narrow lines. The average line width in the Caselli et al. study was $0.33 \mathrm{~km} \mathrm{~s}^{-1}$ for clumps in which no IRAS source is detected. Current chemical models and observations indicate that $\mathrm{NH}_{3}$ and $\mathrm{N}_{2} \mathrm{H}^{+}$are related because $\mathrm{NH}_{3}$ likely forms via pathways linked $\mathrm{N}_{2} \mathrm{H}^{+}$(Aikawa et al. 2005). Therefore, we use the Harju et al. (1993) line widths for $\mathrm{NH}_{3}$ clumps in Orion and Cepheus, known to be large regions of clustered, high-mass star formation for comparison. In addition, we include a comparison with line widths of a sample of ammonia cores presented in Molinari et al. (1996), although we only include only the "Low" sources, a sample they argue has less luminous IRAS sources and more quiescent envelopes and, therefore, are younger than their "High" counterparts. In our sample, we find average line widths of $2.0 \mathrm{~km} \mathrm{~s}^{-1}$ for $\mathrm{N}_{2} \mathrm{H}^{+}, 2.1 \mathrm{~km} \mathrm{~s}^{-1}$ for $\mathrm{C}^{18} \mathrm{O}$, and $2.9 \mathrm{~km} \mathrm{~s}^{-1}$ for CS. Although we see a broad range of line widths in the $\mathrm{N}_{2} \mathrm{H}^{+}$ observations of the dark clouds, the characteristic line widths presented here are generally higher than those of the Harju et al. study and, to a greater extent, the Caselli et al. study, which implies that the objects in this sample are likely not associated with low-mass star formation. However, we find good agreement with the Molinari et al. sample.

For the CS $J=2 \rightarrow 1$ transition, the line widths in the dark cloud sample are narrower than those observed by Plume et al. (1997) in survey of massive star-forming regions, which averaged $4.2 \mathrm{~km} \mathrm{~s}^{-1}$ in this line. However, since Plume et al. surveyed regions known to have undergone massive star formation, it is possible that the current generation of massive stars are injecting additional turbulence into the surrounding environment. Similarly, the Shirley et al. (2003) study observed the CS $J=$ $5 \rightarrow 4$ transition in star-forming cores and found line widths averaging $5.6 \mathrm{~km} \mathrm{~s}^{-1}$. The narrower lines in the dark clouds indicates that they are still relatively quiescent and suggest that they may, indeed, be precursor sites of intermediate or massive star formation.

\section{SUMMARY}

We have identified 41 infrared dark (at $8.8 \mu \mathrm{m}$ ) clouds that are opaque, compact, and associated with UC H II regions using $M S X$ survey data. In order to determine some basic characteristics of these dark clouds, we have mapped emission from $\mathrm{N}_{2} \mathrm{H}^{+}$ $1 \rightarrow 0, \mathrm{CS} 2 \rightarrow 1$, and $\mathrm{C}^{18} \mathrm{O} 1 \rightarrow 0$ using the FCRAO. The morphology and relative strengths of these molecular lines varies dramatically, possibly indicating evolutionary differences and/or the presence of undetected embedded protostar(s). Based on the derived kinematic distances and the simplifying assumption that the cores are optically thin, we have determined average properties: diameter $\langle D\rangle \approx 0.9 \mathrm{pc}$, density $\langle n\rangle \approx 5000 \mathrm{~cm}^{-3}$, and mass $\langle M\rangle \approx 2500 M_{\odot}$. The low-density estimate likely indicates that the dark clouds are clumpy rather than homogeneous. The derived masses, however, are comparable to those derived for a sample of HMPOs. The line widths are larger than those seen in low-mass star-forming cores and larger than in high-mass star-forming cores in Orion and Cepheus. However, they are narrower than the CS line widths seen in regions that are actively forming massive stars. These observations, taken together, suggest that the infrared dark clouds may be the relatively quiescent precursors to intermediate or massive star formation, the so-called "pre-protostellar cores." At present, we do not know if these sources are truly starless. Only by obtaining very sensitive infrared observations can we confirm the starless nature of these cores.

We are grateful to the referee for a very thorough report, which improved this paper. We are grateful to J. Weingartner for providing detailed dust opacity coefficients. This research has made use of the SIMBAD database, operated at CDS, Strasbourg, France.

\section{APPENDIX}

\section{COINCIDENT SOURCES}

The coordinates of each dark region were examined by the Set of Identifications, Measurements, and Bibliography for Astronomical Data (SIMBAD) database in search of associated objects indicative of active star formation (e.g., masers, $I R A S$ sources, radio sources). In most cases, the searches yielded no results, but some revealed objects within one arcminute of the dark region's coordinates. Below, we summarize any associations with the objects for which we present emission maps. We note that whenever coincident sources are found, we provide the offsets relative to the central absorption peak position given in Table 1 .

G09.21-0.22.-There is an IRAS source [IRAS $18038-2105 ; \alpha(\mathrm{J} 2000.0)=18^{\mathrm{h}} 06^{\mathrm{m}} 53^{\mathrm{s}} .1, \delta(\mathrm{J} 2000.0)=-21^{\circ} 04^{\prime} 38^{\prime \prime}$ ] in the vicinity of this absorbing cloud, offset by 0.78 from the center of the dark region.

G09.88-0.11.- This region has an associated $1612 \mathrm{MHz} \mathrm{OH}$ maser that is cited in Blommaert et al. (1994). This source, OH 9.8780.127 , has a position of $\alpha(\mathrm{J} 2000.0)=18^{\mathrm{h}} 07^{\mathrm{m}} 59^{\mathrm{s}} .07, \delta(\mathrm{J} 2000.0)=-20^{\circ} 27^{\prime} 34^{\prime \prime} .3$, which is offset from the center of our region by $1 .^{\prime} 05$. This object may be associated with a circumstellar shell around an evolved star, and the velocities believed to correspond to the 
expanding shells of material are 79.5 and $111.3 \mathrm{~km} \mathrm{~s}^{-1}$, which is not coincident with the velocity of the emission detected here $\left(17 \mathrm{~km} \mathrm{~s}^{-1}\right)$.

G10.59-0.31.-This region contains a radio source, located at $\alpha(\mathrm{J} 2000.0)=18^{\mathrm{h}} 10^{\mathrm{m}} 6^{\mathrm{s}} \mathrm{s} 18, \delta(\mathrm{J} 2000.0)=-19^{\circ} 55^{\prime} 33^{\prime \prime} 11$, according to Zoonematkermani et al. (1990), which is offset from the center of our region by 0.'44.

G12.50-0.22.-There is an IRAS source [IRAS $\left.18197-1812 ; \alpha(\mathrm{J} 2000.0)=18^{\mathrm{h}} 13^{\mathrm{m}} 39^{\mathrm{s}} .0, \delta(\mathrm{J} 2000.0)=-18^{\circ} 11^{\prime} 46^{\prime \prime}\right]$ offset by 0.96 from the absorbing region.

G19.37-0.03.-This region is near a known UC H II region, with a water and methanol maser (Codella \& Felli 1995; Szymczak et al. 2000) also identified in the vicinity $\left[\alpha(\mathrm{J} 2000.0)=18^{\mathrm{h}} 26^{\mathrm{m}} 24.3, \delta(\mathrm{J} 2000.0)=-12^{\circ} 3^{\prime} 46^{\prime \prime}\right]$ offset 0.85 from the absorbing region. The peak velocity of this maser is $26.3 \mathrm{~km} \mathrm{~s}^{-1}$ (Szymczak et al. 2000), which is consistent with the velocity of our measured emission $\left(27 \mathrm{~km} \mathrm{~s}^{-1}\right)$. Molinari et al. (1996) also observed this maser site and designated it as Mol 55.

G30.89+ 0.14. - This region has an associated methanol maser, as described by Szymczak et al. (2000). The maser is located at $\alpha(\mathrm{J} 2000.0)=18^{\mathrm{h}} 47^{\mathrm{m}} 14^{\mathrm{s}} 99, \delta(\mathrm{J} 2000.0)=-1^{\circ} 44^{\prime} 7^{\prime \prime}$.99, which is offset from the center of our region by $0^{\prime} \cdot 99$. Szymczak et al. (2000), using a $6.7 \mathrm{GHz}$ survey, measured the internal velocity of the maser source to be $\approx 105 \mathrm{~km} \mathrm{~s}^{-1}$, the velocity of the peak to be $101.5 \mathrm{~km}$ $\mathrm{s}^{-1}$. This is consistent with one of the velocity components we measured in this object $\left(108 \mathrm{~km} \mathrm{~s}^{-1}\right)$.

G32.01+0.05.-This region has an associated IRAS point source $(18470-0050)$ that is offset from the center of our region by 1 I. $^{\prime} 00$ at $\alpha(\mathrm{J} 2000.0)=18^{\mathrm{h}} 49^{\mathrm{m}} 36^{\mathrm{s}} 6, \delta(\mathrm{J} 2000.0)=-00^{\circ} 46^{\prime} 51^{\prime \prime}$, which coincides with a radio source (Becker et al. 1994). This was confirmed to be a methanol maser (observed in the velocity range between 91 and $102 \mathrm{~km} \mathrm{~s}^{-1}$ ) by van der Walt et al. (1995), which is consistent with the emission we observed at $95 \mathrm{~km} \mathrm{~s}^{-1}$.

G34.74-0.12.-This region has an associated IRAS point source $(18526+0130)$ that is offset from the center of our region by 0.80 at $\alpha(\mathrm{J} 2000.0)=18^{\mathrm{h}} 55^{\mathrm{m}} 10^{\mathrm{s}}, \delta(\mathrm{J} 2000.0)=+1^{\circ} 33^{\prime} 57^{\prime \prime}$.

Aikawa, Y., Herbst, E., Roberts, H., \& Caselli, P. 2005, ApJ, 620, 330

Alves, J. F., Lada, C. J., \& Lada, E. A. 2001, Nature, 409, 159

André, P., Ward-Thompson, D., \& Barsony, M. 2000, Protostars and Planets IV, ed. V. Mannings, A. P. Boss, \& S. S. Russell (Tucson: Univ. Arizona Press), 59

Ashby, M. L. N., et al. 2000, ApJ, 539, L115

Bacmann, A., André, P., Puget, J.-L., Abergel, A., Bontemps, S., \& WardThompson, D. 2000, A\&A, 361, 555

Becker, R. H., White, R. L., Helfand, D. J., \& Zoonematkermani, S. 1994, ApJS, 91, 347

Beichman, C. A., Myers, P. C., Emerson, J. P., Harris, S., Mathieu, R., Benson, P. J., \& Jennings, R. E. 1986, ApJ, 307, 337

Bergin, E. A., \& Langer, W. D. 1997, ApJ, 486, 316

Blommaert, J. A. D. L., van Langevelde, H. J., \& Michiels, W. F. P. 1994, A\&A, 287, 479

Buisson, G., Desbats, L., Duvert, G., Forveille, T., Gras, R., Guilloteau, S., Lucas, R., \& Valiron, P. 2002, Continuum and Line Analysis Single-Dish System Manual (CLASS; Grenoble: IRAM) (http://iram.fr/GS/class/class.html)

Carey, S. J., Clark, F. O., Egan, M. P., Price, S. D., Shipman, R. F., \& Kuchar, T. A. 1998, ApJ, 508, 721

Caselli, P., Benson, P., Myers, P., \& Tafalla, M. 2002, ApJ, 572, 238

Codella, C., \& Felli, M. 1995, A\&A, 302, 521

Egan, M. P., Shipman, R. F., Price, S. D., Carey, S. J., Clark, F. O., \& Cohen, M. 1998, ApJ, 494, L199

Fich, M., Blitz, L., \& Stark, A. 1989, ApJ, 342, 272

Garay, G., \& Lizano, S. 1999, PASP, 111, 1049

Goldsmith, P. 1987, in Interstellar Processes (Dordrecht: Reidel), 51

Harju, J., Walmsley, C. M., \& Wouterloot, J. G. A. 1993, A\&AS, 98, 51

\section{REFERENCES}

Indebetouw, R., et al. 2005, ApJ, 619, 931

Jørgensen, J. K. 2004, A\&A, 424, 589

Lee, C. W., \& Myers, P. C. 1999, ApJS, 123, 233

Lee, J.-E., Bergin, E. A., \& Evans, N. J. 2004, ApJ, 617, 360

Menten, K. M., Pillai, T., \& Wyrowski, F. 2005, IAU Symp. 227, Massive Star Birth: A Crossroads of Astrophysics, ed. R. Cesaroni et al. (Cambridge: Cambridge Univ. Press), 23

Molinari, S., Brand, J., Cesaroni, R., \& Palla, F. 1996, A\&A, 308, 573

Myers, P. C., \& Benson, P. J. 1983, ApJ, 266, 309

Pillai, T., Wyrowski, F., Carey, S. J., \& Menten, K. M. 2006, A\&A, 450, 569

Plume, R., Jaffe, D. T., Evans, N. J., II, Martín-Pintado, J., \& Gómez-González, J. 1997, ApJ, 476, 730

Rathborne, J. M., Jackson, J. M., \& Simon, R. 2006, ApJ, 641, 389

Shirley, Y. L., Evans, N. J., II, Young, K. E., Knez, C., \& Jaffe, D. T. 2003, ApJS, 149, 375

Sridharan, T. K., Beuther, H., Saito, M., Wyrowski, F., \& Schilke, P. 2005, ApJ, 634, L57

Szymczak, M., Hrynek, G., \& Kus, A. J. 2000, A\&AS, 143, 269

Tafalla, M., Myers, P. C., Caselli, P., Walmsley, C. M., \& Comito, C. 2002, ApJ, 569, 815

van der Walt, D. J., Gaylard, M. J., \& MacLeod, G. C. 1995, A\&AS, 110, 81 Weingartner, J. C., \& Draine, B. T. 2001, ApJ, 548, 296

Williams, S. J., Fuller, G. A., \& Sridharan, T. K. 2004, A\&A, 417, 115

Wood, D. O. S., \& Churchwell, E. 1989, ApJS, 69, 831

Zinnecker, H., McCaughrean, M. J., \& Wilking, B. A. 1993, in Protostars and Planets III (Tucson: Univ. Arizona Press), 429

Zoonematkermani, S., Helfand, D. J., Becker, R. H., White, R. L., \& Perley, R. A. 1990, ApJS, 74, 181 